\title{
Obsolescence of Durable Goods and Optimal Purchase Timing
}

\author{
Ennio Stacchetti \\ Department of Economics, New York University \\ 19 West Fourth Street, 6th floor \\ New York, NY 10012 \\ E-mail: ennio@nyu.edu \\ Dmitriy Stolyarov \\ Department of Economics, University of Michigan \\ 611 Tappan Ave \\ Ann Arbor, MI 48109-1220 \\ E-mail: stolyar@umich.edu
}

July 9, 2015 


\begin{abstract}
We study a model with a durable good subject to periodic obsolescence and analytically characterize the optimal purchasing policy. The key result is that consumers optimally synchronize new purchases with the innovation cycle. The model simultaneously explains coordinated adoption without invoking network effects and provides a theoretical underpinning for a diffusion curve that features a temporary adoption slowdown. ${ }^{1}$
\end{abstract}

Keywords: Obsolescence, Durable goods, Technology adoption.

\footnotetext{
${ }^{1}$ Both authors gratefully acknowledge the financial support from the National Science Foundation (NSF) SES-0241640. We thank Marco Bassetto, Ricardo Caballero, Boyan Jovanovic, Alessadro Lizzeri and Richard Rogerson for insightful comments and two anonymous referees for their help in improving the paper.
} 


\section{Introduction}

The arrival of better products at the same price is the major reason for depreciation in markets with technological innovation. Since much of this innovation is incorporated in new durables, modeling obsolescence of durable goods is vital for our understanding of technology adoption. Our goal is to characterize the aggregate demand for durables in a dynamic model of consumer choice that captures the essential distinctions between obsolescence and physical depreciation.

Obsolescence of a durable occurs with mere passage of time, typically because superior substitutes become available at the same price. By contrast, physical depreciation depends on utilization intensity (or the good's decay with physical age) specific to an individual unit. Thus, obsolescence and utilization are two distinct depreciation channels. Their aggregate effects are distinct, as well: while physical depreciation is idiosyncratic and its aggregate effects are likely smooth, obsolescence caused by innovation affects all durables within a market. Moreover, technological events that cause obsolescence may be predictable: major innovation episodes can be anticipated, especially when the introduction of new products is periodic. For some goods, such as automobiles, redesigned models do appear periodically, every 4 or 5 years. Even when obsolescence is not deterministic, obsolescence episodes are typically not independent events either. Innovation processes naturally have hazard rates that are negligible immediately after an innovation; after all, no one expects a new generation of products to appear immediately after the introduction of a new model. Therefore, we think that an innovation process with predictable, discrete jumps captures the main features of obsolescence that are distinct from physical depreciation. ${ }^{2}$

Our analysis builds on the basic idea that consumer expectations about the timing of future innovations affect current purchasing behavior. ${ }^{3}$ Suppose that individual units are expected to depreciate abruptly at some future date. Consumers who purchase their durables

\footnotetext{
${ }^{2}$ In reality, obsolescence patterns have both discrete and continuous elements, but markets in which discrete obsolescence is likely to be important are commonplace. The literature typically associates periodic obsolescence with a monopolistic producer whose timing of product introduction is a strategic variable (e.g., Swan, 1972; Rust, 1986; Fishman and Rob, 2000). Our focus is on a different set of markets, where major innovations affect all the producers, but they are infrequent due to technological constraints rather than strategic reasons. These markets include several (overlapping) categories. (1) Markets in which new products have a different format or standard. Format switching is typical for data recording or storage devices, such as disk drives, camcorders and digital cameras. (2) Goods that depend on a "bottleneck" (lagging) technology. For example, power supply has been a constraining factor in adding new features to many portable electronic devices. (3) Markets in which technological constraints are imposed by periodically changing government regulation, such as cellular communications.

${ }^{3}$ The idea of expectations-driven demand is similar in spirit to frameworks featuring deterministic output cycles: Shleifer (1986) and Francois and Lloyd-Ellis (2003) demonstrate how coordination of innovation dates across producers can arise from agents' rational expectations about the timing of economic booms and give rise to aggregate deterministic output cycles.
} 
just before this date will enjoy a lower service flow than those who buy soon after. Hence, consumers have an incentive to buy a durable only when the design is sufficiently new and is not about to change soon. Thus, demand for new durables should drop some time prior to the dates when the new models become available. These anticipatory drops in demand have been noted for DVD players (Dranove and Gandal, 2003) and large-screen TVs (Shapiro and Varian, 1999, p. 15). In automobiles, regular timing of model year changes induces strong seasonal fluctuations in auto sales (Cooper and Haltiwanger, $1993 \mathrm{a}, \mathrm{b})$.

We study an economy with a durable and a non-durable good and a large number of heterogeneous consumers. The durable good in our model represents a fairly narrow consumption category; hence, the assumption that innovation is periodic seems appropriate. Then, the non-durable category encompasses all options of deriving utility from expenditure outside of the durable market. The main feature that makes our framework distinct from a standard $(s, S)$ model of durable replacement is the periodic (rather than continuous) arrival of better models of the durable. The periodic nature of model changes introduces a mixture of discrete (e.g., whether to buy the current model or wait for the next one) and continuous (e.g., when to buy the current model) choice variables and makes the replacement problem difficult to analyze. Nevertheless, we develop a special solution methodology that does not rely on marginal conditions and are able to solve analytically for the optimal consumption paths of individuals.

Our basic model features periodic innovation dates that are perfectly anticipated. One key property of the optimal solution is the no-delay result (Theorem 2): all consumers who purchase a particular model of the durable find it optimal to do so simultaneously, at the time when this model is first introduced. The timing of purchases depends on the interest rate and the (endogenous) marginal utility of wealth. If a consumer is buying the current model of the durable, and the interest rate is zero, then she is clearly better off buying without delay and getting the highest possible service flow from the new model. However, as the interest rate increases, consumers may prefer to buy in the middle of the design cycle, despite the loss of service flow. We show that purchasing a durable in the middle of the design cycle is never optimal because any consumer can be made better off by either buying the current model without delay or by buying some future model without delay.

Two unique implications derive from the no-delay result. First, it gives rise to a new mechanism for demand coordination that is not dependent on network effects, externalities or strategic complementarities. Previous literature that sought to understand simultaneous technology adoption stresses a different coordination mechanism based on positive externalities, such as information spillovers (Bannerjee, 1992), learning by doing (Jovanovic and Lach, 1989) and consumption externalities (Farrell and Saloner, 1985). The policy implications of 
the two mechanisms are distinct: adoption timing in our model is efficient, whereas in a setting with externalities, it is inefficiently slow. ${ }^{4}$

Second, the coordination mechanism that we identify in the basic model allows a more detailed understanding of empirical technology diffusion curves. The basic argument can be generalized to a setting in which model arrival dates are random, but the innovation hazard rate is negligible immediately after a new model introduction. When the hazard rate is initially negligible, consumers who purchase the durable early enjoy a longer time without obsolescence. Consumers then optimally separate themselves into two groups: early adopters, who act (almost) immediately, and late adopters, who choose to purchase the good with a long delay. Early adopters purchase the good at a higher price but are less exposed to the risk of obsolescence. By contrast, late adopters, who face a higher risk of obsolescence, find it optimal to wait until the price of the good falls. Since all consumers decide to act either early or late, no one purchases the good in the middle of its design cycle, and its diffusion curve reaches a temporary "plateau." 5

Recent empirical results on diffusion curves are generally consistent with diffusion slowdown after the initial burst in demand. For example, Comin et al. (2006) outline the general characteristics of technology adoption patterns and conclude that "once the intensive margin is measured, technologies do not diffuse in a logistic way." In particular, for many technologies, a slowdown in the rate of diffusion follows the initial burst of adoption activity (see also Comin and Hobijn (2010, Figures 2, 3)). This is the opposite of the S-shaped, or logistic, diffusion pattern, where the adoption rate is highest in the middle of the diffusion process.

Predictable obsolescence patterns have dramatic effects on purchase timing: the no-delay property, for instance, restricts the relevant choices of durable purchase dates to be discrete. This gives rise to a non-standard consumption smoothing mechanism: some consumers prefer a fixed (discrete) replacement frequency, while others alternate between two discrete holding periods from time to time. For the latter group, optimality requires that the marginal utility of wealth (as well as the non-durable consumption) be independent of purchase timing choices or the wealth level. Interestingly, if such consumers receive an unexpected windfall, they will not change their non-durable consumption but will, instead, spend the entire amount of

\footnotetext{
${ }^{4}$ As a specific example of a technology adoption subsidy, the Senate Commerce Committee approved a Digital TV bill that provided a $\$ 1.5$ billion subsidy to consumers to facilitate the switch to HDTV (Source: US Senate Committee on Commerce, Science and Transportation Press release, Dec 21, 2005). Our model suggests that some consumers were optimally waiting for the future generations of digital TV models, and the subsidy was not needed to incentivize them.

${ }^{5}$ Balcer and Lippman (1984) analyze the technology adoption problem under uncertainty with timevarying innovation arrival rate. They find that expected arrival of a better technology limits the total number of adopters, but makes them act fast. While our work shares a similar basic idea, we solve a more general (and a more challenging) problem with a budget constraint and highlight the features of the diffusion curve that are due to an uneven rate of obsolescence.
} 
additional wealth on future durable purchases. The last result is reminiscent of what Leahy and Zeira (2005) term "insulation effect": the windfall is entirely absorbed by changes in durable purchase timing, and the non-durable consumption is, thus, insulated from the wealth shock. The insulation effect is not universal in our model, however. The other group of consumers, who optimally use a fixed holding frequency, will spend the entire windfall on non-durable consumption and not change their durable purchase timing.

Our work complements two strands of macroeconomic literature that study obsolescence and integrates their previously separate sets of assumptions about the innovation process. One such strand of the literature (see Boucekkine et al., 2011 for a comprehensive literature review) is macroeconomic models that derive from Solow's (1960) vintage capital framework. The key assumption shared with our model is innovation embodied in durable goods. Another strand uses Schumpeterian models of creative destruction, particularly frameworks that follow the seminal quality ladder model (Grossman and Helpman, 1991). The shared feature with our model is the sector-level production technology subject to repeated, abrupt obsolescence. There is no embodiment in the quality ladder framework, however: capital is general rather than technology-specific. The distinct implications in our model are due largely to the combination of the previously separate embodiment and creative destruction assumptions. Our analysis provides a detailed description of the consumer side of the economy in the presence of lumpy durable goods, and, as such, it is complementary to the macroeconomic models of obsolescence that focus on the production side.

The remainder of the paper is organized as follows. Section 2 describes the model. Section 3 separately solves the special case of the optimal consumption problem for durables and non-durables. Section 4 characterizes the general case solution. Section 5 discusses the aggregate implications of our main results in the context of innovation driven by general purpose technologies. In Section 6, we analyze the model with stochastic innovation and derive the diffusion curve. Section 7 concludes.

\section{Model}

We consider a dynamic economy with two goods, a durable and a non-durable good, and a continuum of agents that differ in their permanent income $y \in[y, \bar{y}]$. Incomes are given exogenously and stay constant over time.

Goods, TECHNOLOGy And PREFEREnCEs: The durable good is indivisible and is produced with a constant returns-to-scale technology that uses $p_{0}$ units of the non-durable good for each unit of the durable good. New durables (new models) are introduced regularly into the market at times $\tau \in \mathbf{N}=\{0,1, \ldots\}$. Without loss of generality, we have normalized to 1 
the length of a design cycle. We refer to the durable introduced at time $\tau$ as "model $\tau . "$ Obsolescence is the only form of depreciation in our model. ${ }^{6}$ Suppose that a model $\tau \geq 0$ provides a constant service flow $z_{\tau}=e^{g \tau}$ for the duration of its useful life in the interval $[\tau, \tau+T)$, where $T$ is a positive integer. In other words, the durable becomes useless when it falls $T$ or more models behind the state-of-the-art good. A useless durable provides service flow equal to 1 . Notice that $\tau+T$ acts as an "expiration date" for any durable of model $\tau$, regardless of whether it was produced at date $\tau$ or some later date $t>\tau$.

The expiration date assumption has both technical and substantive content. Technically, our solution methodology requires an upper bound on the holding time for the durable good, and the expiration date is a simple way to impose such a bound (see, in particular, Theorem 1). Substantively, the assumption derives from the Schumpeterian concept of innovation and states that recurring arrivals of superior models eventually cause creative destruction (here, every $T$ generations of products). This assumption captures the fact that the useful life of many durables is limited not by the extent of physical wear and tear, but by eventual arrival of far superior models. ${ }^{7}$ For example, a sundial can stay physically functional indefinitely, but it might fall into disuse when consumers gain access to a quartz watch. In this example, the arrival date of a quartz watch acts as the expiration date for the sundial.

Consumers: Consumers are infinitely-lived and have a (common) discount rate $\rho$ and a (common) separable flow utility function $f(\tau, c)=\ln z_{\tau}+u(c)$, where $\tau$ is the durablegood model and $c$ is the consumption flow for the non-durable (note that a useless durable provides utility of zero). Durable goods are perfect substitutes, and each agent consumes, at most, one unit (additional units provide no utility). We think of the non-durable good as money for the consumption of other goods, and of $u$ as an indirect utility function. We assume that $u^{\prime}>0, u^{\prime \prime}<0, u^{\prime}(0)=\infty$.

The consumers can borrow and lend, but there are no secondary markets for used durables (the latter assumption is relaxed in Section 4.2).

PRICES: Since the production technology has constant returns to scale, the price ratio of the durable good to the non-durable good is equal to a constant $p_{0}$ at all times. We will assume that the interest rate is fixed and equal to the discount rate: $r(t)=\rho$ for all $t \geq 0$. Therefore, we perform a partial-equilibrium analysis. We think of the market for durables as being a small part of the aggregate economy and, hence, ignore the effect of durable demand

\footnotetext{
${ }^{6}$ Adding deterministic physical depreciation will not affect our results. See the discussion following Theorem 2 .

${ }^{7}$ In a richer model, using the durable might involve a rising fixed cost (e.g., labor cost of maintenance), and $T$ would arise endogenously as the age when the fixed cost of maintaining the good exceeds its utility. For example, Johansen (1959) proposed a vintage capital model with fixed labor costs that features an endogenous range of active vintages.
} 
on the interest rate. Our choice of interest rate is consistent with stationary equilibria. In a general equilibrium model in which income (resource) flow and production technology are constant over time, a stationary equilibrium would imply a constant interest rate equal to the discount rate. If $q(t)$ and $p(t)$ denote, respectively, the prices of the non-durable and durable goods at time $t$, our assumption of a constant interest rate implies that $q(t)=e^{-\rho t}$ and $p(t)=p_{0} q(t)$ for all $t \geq 0$, where we have normalized so that $q(0)=1$. Define the total discount rate for one period as $\beta=e^{-\rho}$.

Equivalent utility Function: The model features a time trend in durable quality, and it is convenient to formulate an equivalent problem in detrended variables. Let $\alpha=\{0, \ldots, T\}$ denote the technological age of a durable good - i.e., the number of new models introduced since it was produced. Because all useless goods are the same, we make no distinction between durables whose technological age exceeds $T$, so all of them have $\alpha=T$. At time $t$, the state-of-the-art model is $\lfloor t\rfloor$, where $\lfloor t\rfloor$ is the largest integer less than or equal to $t$. Model $\lfloor t\rfloor$ delivers flow utility $g\lfloor t\rfloor$, and a model of age $\alpha$ provides utility $g(\lfloor t\rfloor-\alpha)$. The utility differential between model age $\alpha$ and the state-of-the-art model depends only on $\alpha$ (and not on $t$ ) and equals $-g \alpha$. Accordingly, we can define a normalized ${ }^{8}$ utility differential

$$
x_{\alpha}=g(T-\alpha) \text { for } \alpha=0, \ldots, T \text {, }
$$

so that $x_{\alpha}=x_{0}-g \alpha$. Proposition 1 below states that a consumer's ranking of different consumption paths depends only on the utility of her durable compared to the state-of the-art model.

Proposition 1: Let $\alpha(t)$ and $c(t)$ be two measurable functions representing the consumption trajectory of a consumer (where $\alpha(t)$ is the technological age of the durable being consumed at time $t$ ). Then, the discounted lifetime utility for this trajectory is

$$
U(\alpha, c)=\int_{0}^{\infty} e^{-\rho t} f(\lfloor t\rfloor-\alpha(t), c(t)) d t=K+\int_{0}^{\infty} e^{-\rho t} \hat{f}(\alpha(t), c(t)) d t
$$

where $K$ is a constant and

$$
\hat{f}(\alpha(t), c(t))=x_{\alpha(t)}+u(c(t))
$$

Proof: See Appendix.

For the rest of the paper, we will use the equivalent utility function in (2) and refer to $\hat{f}$

\footnotetext{
${ }^{8}$ It is convenient to choose a normalization where the useless good has $x_{T}=0$, which is why we add a constant $g T$ to the utility differential in (1).
} 
and $x_{\alpha}$ simply as "utility." 9

It is easy to see that any delay in purchasing the current model reduces its discounted utility. Suppose that a new model $\tau$ is purchased at date $\tau+d, d$ periods after it first appears. Let $R$ be the holding time for the durable, and let $X_{d, R+d}$ denote the total discounted utility of a model held from age $d$ until age $R+d$ :

$$
X_{d, R+d}=\int_{d}^{\min \{T, R+d\}} e^{-\rho(t-d)} x_{\alpha(t)} d t .
$$

The utility of the durable $x_{\alpha}$ drops at pre-determined dates $\{\tau+1, \tau+2, \ldots, \tau+T\}$ independent of $d$; hence any delay reduces utility: $X_{d, R+d}<X_{0, R}$, all $d>0$. In other words, technological aging occurs with the mere passage of time, so the model purchased with a delay experiences some depreciation even before it is used. This property is specific to obsolescence: if depreciation were, instead, a result of physical wear alone, there would be no depreciation before use, and consumers could enjoy the full utility $X_{0, R}$ from any new model regardless of its purchase date.

Consumer problem: Given her initial state $\left(\alpha_{0}, w\right)$, where $\alpha_{0} \in\{1, \ldots, T\}$ is the age of her endowed durable and $w$ is her total wealth, a consumer chooses a sequence of durable purchase dates and a non-durable consumption path to maximize her discounted lifetime utility, $U(\alpha, c)$, subject to a lifetime budget constraint. An agent's current wealth, $w$, is equal to her initial assets plus the present discounted value of future earnings $y / \rho$.

Since the agent's utility is additively separable, the optimal consumption problem can be solved in two stages. First, choose how to divide the wealth into budgets $w-b$ and $b$ that will be used in nondurable and durable consumption. Second, given budgets $w-b$ and $b$, select optimal consumption schedules for the nondurable and durable goods.

When $r(t) \equiv \rho$, the optimal nondurable consumption is constant over time. Indeed, the (necessary and sufficient) first-order condition for non-durable consumption is, in this case, $e^{-\rho t} u^{\prime}(c(t))=\lambda e^{-\rho t}$ for all $t$, where $\lambda>0$ is the Lagrange multiplier on the budget constraint. This implies that $c(t)=c(0)$ for all $t>0 .{ }^{10}$

Let $\hat{u}(c)$ be the discounted non-durable consumption utility over one period (of length 1) in which a consumer spends optimally a budget $c$ for the period. Budget $c$ affords the

\footnotetext{
${ }^{9}$ The main results in this paper do not depend on $x_{\alpha}$ being linear in $\alpha$. The analysis of the model is unchanged if the consumer's utility function is of the form (2) and $\left\{x_{\alpha}\right\}$ is any decreasing sequence with $x_{T}=0$.

${ }^{10}$ When $\rho>r$, the optimal $c(t)$ is falling in $t$. In this case, a consumer optimally front-loads her consumption and finances it by borrowing against her future income early in life. Similarly, she will replace durables more frequently early on. The opposite would occur when $\rho<r$ : consumers will choose to postpone consumption and accumulate savings to finance it.
} 
constant instantaneous consumption flow $\kappa c$, where $\kappa=\rho /(1-\beta)$. Accordingly,

$$
\hat{u}(c)=\int_{0}^{1} e^{-\rho t} u(\kappa c) d t=u(\kappa c) / \kappa
$$

Given a total budget $w-b$ for nondurables, the consumer optimally spends $c=(w-b)(1-\beta)$ per period on nondurables and gets a total discounted utility of $\hat{u}(c) /(1-\beta)$. Note that $b=w-c /(1-\beta)$.

Let $V_{\alpha}(b)$ denote the optimal durable consumption utility of a consumer that is endowed with a good of age $\alpha$ and spends a total budget $b$ on durables. Then, the budget allocation problem of an agent with initial state $(\alpha, w)$ is

$$
U_{\alpha}(w)=\max _{b \in[0, w]} \frac{\hat{u}((1-\beta)(w-b))}{1-\beta}+V_{\alpha}(b)
$$

In Section 4, we explicitly construct the functions $V_{\alpha}, \alpha \in\{1, \ldots, T\}$ and obtain the full solution for problem (4).

Solution methodology: Given a budget $b$, solving for the optimal durable consumption path $\alpha(t)$ requires finding a sequence of dates $\left\{t_{k}\right\}$ when the durable is replaced. This is difficult because the utility of the durable, $x_{\alpha(t)}$, jumps discontinuously every time the durable is replaced or a new model arrives. Thus, the optimal $\left\{t_{k}\right\}$ is not characterized by standard marginal conditions. To tackle this non-standard problem, we first restrict attention to a class of feasible durable purchasing rules (that we call no-delay policies) by which the consumer buys new durables only at dates $t_{k} \in \mathbf{N}$ when new models appear. We are able to characterize the best no-delay policy analytically, as a solution to an integer programming problem (see Theorem 1). We then show that the best no-delay policy is also optimal for the problem where the consumer can make durable purchases at any time $t_{k} \in \mathbf{R}_{+}$(see Theorem 2 ). The next section illustrates the steps involved in constructing the best no-delay policy for the special case $T=1$. Section 4 then goes on to describe our main results for the general case $T>1$.

\section{Special case $T=1$}

We start with an example that illustrates why the optimal policy might belong to the nodelay class. Next, we show how to construct the best no-delay policy (which, in the end, will coincide with the optimal policy) for $T=1$, and we point out the features that the optimal policy has in common with the general case solution derived in Section 4.

Assume that $\beta \in(1 / 2,1)$ and $T=1$. Hence, a new model provides a constant utility 
$x_{0}>0$ during its first period and, subsequently, a utility of 0 . Consider a consumer that has a budget $b$ for durable consumption and a good of age $\alpha_{0}=1$. If the consumer replaces the durable every period as soon as the new models arrive, including period 0 , she spends $p_{0} /(1-\beta)$ and gets a constant flow utility of $x_{0}$ and a lifetime discounted utility of $x_{0} / \rho$. If she skips model $\tau=0$, instead, and purchases every subsequent model, she spends $\beta p_{0} /(1-\beta)$ and obtains a total utility of $\beta x_{0} / \rho$. Now, compare two different ways of spending a budget $b$ in the intermediate range $b \in\left(\beta p_{0} /(1-\beta), p_{0} /(1-\beta)\right)$.

With a budget $b$, the consumer cannot replace the durable every period but can afford the following two durable purchasing policies. Policy $\mathrm{A}$ is to make the initial purchase of the durable with a delay $d \in(0,1)$ in period 0 and then to replace the good every period as soon as new models arrive. The delay is exactly such that policy A spends budget $b$ :

$$
b=p_{0} e^{-\rho d}+\frac{\beta p_{0}}{1-\beta}=\frac{p_{0}}{1-\beta}-p_{0}\left(1-e^{-\rho d}\right) .
$$

For $d \in[0,1]$, policy $\mathrm{A}$ allows consumers to spend a subset of intermediate budgets $b \in$ $\left[\frac{p_{0}}{1-\beta}-p_{0}(1-\beta), \frac{p_{0}}{1-\beta}\right]$. In contrast, policy B purchases a new model in period 0 without delay and then skips a (possibly infinite) sequence of future models $\left\{\tau_{k}\right\}$ such that

$$
\sum_{k=1}^{\infty} \beta^{\tau_{k}}=1-e^{-\rho d} .
$$

That is, the total budget savings from skipping future models amount to $p_{0}\left(1-e^{-\rho d}\right)$. Therefore, policy A and policy B spend exactly the same budget $b$. However, policy B has higher total utility:

$$
\begin{gathered}
v_{B}=\frac{x_{0}}{\rho}-\sum_{k=1}^{\infty} \beta^{\tau_{k}} X_{0,1}=\frac{x_{0}}{\rho}-\left(1-e^{-\rho d}\right) X_{0,1} \\
>\frac{x_{0}}{\rho}-\frac{1-e^{-\rho d}}{1-\beta} X_{0,1}=v_{A} .
\end{gathered}
$$

The total utility of policy B is higher because any delay in purchasing the current model reduces its lifetime service flow without generating a corresponding savings in budget. Indeed, a consumer who buys model $\tau$ without delay spends $p_{0} \beta^{\tau}$ and obtains the present value of $\beta^{\tau} \frac{x_{0}}{\rho}(1-\beta)$. Her utility per dollar spent on durables is, therefore, $\frac{x_{0}}{p_{0}} \frac{1-\beta}{\rho}$, independent of $\tau$. When the consumer buys the same model with a delay $d$, she spends $e^{-\rho d} p_{0} \beta^{\tau}$ and obtains a present value of $\beta^{\tau} x_{0} \frac{e^{-\rho d}-\beta}{\rho}$. In this case, her utility per dollar is $\frac{x_{0}}{p_{0}} \frac{1-\beta e^{\rho d}}{\rho}<\frac{x_{0}}{p_{0}} \frac{1-\beta}{\rho}$. In the extreme case, in which the consumer buys model $\tau$ one instant before the next model 
is introduced (so $d=1$ ), the utility per dollar is 0 . The example suggests that policies with delays can be dominated by no-delay policies spending the same budget. We formalize and generalize this argument in Theorem 2.

Now, construct the best no-delay policy for any $b$. Let $\left\{\tau_{k}\right\}$ be a sequence of models purchased with no delays. The value and budget of any such no-delay policy can be represented as

$$
v=\sum_{k=1}^{\infty} \beta^{\tau_{k}} X_{0,1}, \quad b=\sum_{k=1}^{\infty} \beta^{\tau_{k}} p_{0} .
$$

Combining the above expressions and noting that the maximum attainable durable utility is $\frac{x_{0}}{\rho}$,

$$
v=V_{1}(b)=\left\{\begin{array}{ll}
\frac{X_{0,1}}{p_{0}} b, & b \in\left[0, \frac{p_{0}}{1-\beta}\right], \\
\frac{x_{0}}{\rho}, & b \geq \frac{p_{0}}{1-\beta}
\end{array} .\right.
$$

The optimal decision rule $\delta_{1}^{*}(b) \in\{0,1\}$ specifies whether to replace $(\delta=1)$ the $\alpha=1$ good or keep it $(\delta=0)$ for one more period. The decision rule $\delta_{1}^{*}(b)$ can be obtained as a solution to the Bellman equation

$$
V_{1}(b)=\max \left\{X_{0,1}+\beta V_{1}\left(\frac{b-p_{0}}{\beta}\right), \beta V_{1}\left(\frac{b}{\beta}\right)\right\}
$$

Equation (6) describes a discrete choice: replace or keep a good $\alpha=1$. If the consumer replaces $(\delta=1)$, she collects utility $X_{0,1}$ in the current period and enters the next period with a good of age $\alpha=1$ and a durable budget $\frac{1}{\beta}\left(b-p_{0}\right)$. If the consumer keeps $\alpha=1$ good for another period, she gets zero utility in the current period and enters the next period with a durable budget $\frac{b}{\beta}$. Figure 1 (left panel) depicts the two functions in the right-hand side of the Bellman equation (6) and the corresponding optimal decision rule for the case $\beta \geq \frac{1}{2}$. When $b<p_{0}$, it is not feasible to replace the good, so $\delta=0$. When $b \in\left[p_{0}, \frac{\beta p_{0}}{1-\beta}\right]$, the consumer is indifferent between keeping and replacing: $\delta=\{0,1\} .{ }^{11}$ This means that there is more than one policy $\left\{\tau_{k}\right\}$ that can spend a budget $b$ in the indifference range, but all such policies have the same value $A_{1} b$. When $b>\frac{\beta p_{0}}{1-\beta}$, the optimal decision is to replace $(\delta=1)$. Theorem 1 formally proves that the value function (5) solves (6) and generalizes the argument for $T>1$.

To summarize, consumers who can afford to replace the good every period $\left(b \geq \frac{p_{0}}{1-\beta}\right)$ optimally do so. We say that they are following a 1-fixed rule. This terminology means that

\footnotetext{
${ }^{11}$ Assumption $\beta \geq 1 / 2$ is essential for the value function $V_{1}(b)$ in (5) to solve $(6)$. When $\beta<1 / 2$, it is no longer possible to spend every budget $b \in\left[0, p_{0} /(1-\beta)\right]$ following a 1-flexible rule. Intuitively, when $\beta$ is low, the wealth accumulation rate per period is high. Some consumers who start off with a 1-flexible rule eventually become wealthy enough to switch to a 1-fixed rule. The discussion after Theorem 1 provides more detail.
} 
they maintain a fixed holding cycle of 1 period. By contrast, consumers with $b<\frac{p_{0}}{1-\beta}$ follow what we call a 1-flexible rule. They hold each durable for at least one period and can skip one or more models between subsequent replacements. The durable replacement frequency is, therefore, irregular. To illustrate, suppose that the starting budget is $0<b<p_{0}$, so it is optimal to skip the first model. Each period that the new model is skipped, the budget grows by a factor $\frac{1}{\beta}$. Eventually, the budget grows enough to exceed $p_{0}$ and make it (weakly) optimal to purchase the first new model

$$
\tau_{1}=\min _{n}\left\{n:\left(\frac{b}{\beta}\right)^{n}>p_{0}\right\} .
$$

The new purchase reduces the budget to $\frac{b-p_{0}}{\beta}$ next period, and the skipping phase starts again. Notice that each time the flexible-rule consumer revisits the state $\alpha=1$, the current $b$ is different (as it depends on her past replacement history). Consequently, the intervals between replacements are irregular, and the demand for new goods by flexible-rule consumers follows a seemingly erratic, rather than periodic, pattern.

The optimal value function obtained in (6) makes it easy to solve the budget allocation problem (4) for the special case $T=1$ :

$$
U_{1}(w)=\max _{b \in[0, w]} \frac{\hat{u}((1-\beta)(w-b))}{1-\beta}+V_{1}(b) .
$$

Consumers following the 1 -flexible rule choose a budget $b \in\left(0, \frac{p_{0}}{1-\beta}\right)$, and, for them, problem (7) has an interior solution $c_{1}^{*}(w)$ obeying the first-order condition

$$
\hat{u}^{\prime}\left(c_{1}^{*}(w)\right)=\frac{X_{0,1}}{p_{0}} .
$$

Hence, every 1-flexible-rule consumer has the same non-durable consumption level

$$
c_{1}^{*}(w)=c_{1}=\left[\hat{u}^{\prime}\right]^{-1}\left(\frac{X_{0,1}}{p_{0}}\right)
$$

irrespective of their $w$. Consumers whose wealth is not enough to afford $c_{1}$ choose a corner solution with $b=0$. At the other end of the wealth interval, consumers who can afford a durable budget $\frac{p_{0}}{1-\beta}$ and a non-durable consumption level of at least $c_{1}$ choose the other corner solution, $b=\frac{p_{0}}{1-\beta}$. Summarizing, optimal non-durable consumption is a piecewise 
linear function

$$
c_{1}^{*}(w)= \begin{cases}(1-\beta) w, & w<\frac{c_{1}}{1-\beta} \\ c_{1}, & w \in\left[\frac{c_{1}}{1-\beta}, \frac{c_{1}+p_{0}}{1-\beta}\right] . \\ (1-\beta)\left(w-p_{0}\right), & w>\frac{c_{1}+p_{0}}{1-\beta}\end{cases}
$$

depicted in Figure 1 (right panel).

The analysis of the special case informs the solution methodology for the general case, as the no-delay replacement policies and the associated fixed and flexible rules also arise when $T>1$.

\section{General case}

We solve problem (4) in the general case $T>1$ using a guess-and-verify strategy. Based on the analysis in Section 3, we guess that the optimal durable purchasing policy belongs to a class of no-delay policies with $\tau_{k} \in \mathbf{N}$ for any $k$. Theorem 1 derives the optimal value function within the restricted class of no-delay policies. Theorem 2 verifies that the unrestricted optimal policy, indeed, belongs to the no-delay class. In other words, it turns out that Theorem 1 fully characterizes the solution to the durable replacement problem.

No-DELAY OPTIMAL POLICY: We now construct the optimal value function $V_{\alpha}(b)$ for the general case $T>1$. The main difference from the special case is that consumers can replace their goods before they reach age $T$. We will show that the optimal solution features $T$ distinct fixed rules with holding periods $R \in\{1, \ldots, T\}$ and $T$ distinct flexible rules by which consumers episodically switch between holding periods $R$ and $R+1$.

A no-delay durable purchasing policy is a sequence of decisions $\delta=\left\{\delta_{t}\right\}_{t \geq 0}$, where $\delta_{t}=1$ if the consumer buys a new unit in period $t$ and $\delta_{t}=0$ if she keeps the old unit. To make notation more compact, define $i \oplus j=\min \{i+j, T\}$ and $i \ominus j=\max \{i-j, 0\}$ for any $i, j \in \mathbf{N}$. Given an initial durable of age $\alpha$, a purchasing policy determines the age of the durable consumed in every period $t \geq 0$ recursively, as follows: $\alpha_{t}=0$ if $\delta_{t}=1$ and $\alpha_{t}=\alpha_{t-1} \oplus 1$ if $\delta_{t}=0$, where we define $\alpha_{-1} \equiv \alpha-1$ (and allow for $\alpha_{-1}=-1$ if $\alpha=0$.)

The total discounted utility from the consumption of a durable of age $\alpha$ over one period is $\hat{x}_{\alpha} \equiv x_{\alpha} / \kappa$. Hence, the optimization problem of an agent that initially has a good of age 
$\alpha$ and durable budget $b$ is

$$
\begin{aligned}
& V_{\alpha}(b)=\max \sum_{t=0}^{\infty} \beta^{t} \hat{x}_{\alpha_{t}} \\
& \text { s.t. } \quad \delta_{t} \in\{0,1\} \text { and } \alpha_{t}=\left\{\begin{array}{ll}
0, & \delta_{t}=1, \\
\alpha_{t-1} \oplus 1, & \delta_{t}=0
\end{array}, t \geq 0\right. \\
& \quad b=p_{0} \sum_{t \geq 0} \beta^{t} \delta_{t} .
\end{aligned}
$$

After introducing some additional notation, we are able to solve the integer programming problem above using a direct geometric argument.

Definition: For each $R=1, \ldots, T$, a policy $\delta$ that replaces the durable every time it reaches age $R$ is called an $R$-fixed rule. That is, $\delta$ is an $R$-fixed rule if for all $t, \delta_{t}=1$ if and only if $\alpha_{t-1} \geq R-1$. A $(T+1)$-fixed rule is to never replace the durable: $\delta_{t}=0$ for all $t$.

The total utility derived from an $R$-fixed rule depends on the initial age $\alpha$ of the durable that the consumer is endowed with. Let $X_{\alpha, R}$ denote the total discounted utility from holding a durable from age $\alpha$ until age $R$ :

$$
X_{\alpha, R}= \begin{cases}\sum_{t=\alpha}^{R-1} \beta^{t-\alpha} \hat{x}_{\alpha} & \alpha<R \\ 0 & \alpha \geq R\end{cases}
$$

For $R \leq T$, the value of following the $R$-fixed rule starting with $\alpha=T$ equals $v_{T, R}=$ $X_{0, R} /\left(1-\beta^{R}\right)$, and its corresponding budget is $b_{T, R}=p_{0} /\left(1-\beta^{R}\right)$. The value and budget of the $(T+1)$-fixed rule are both zero.

Construct a piecewise linear function by joining the adjacent points $\left(b_{T, R+1}, v_{T, R+1}\right)$ and $\left(b_{T, R}, v_{T, R}\right)(1 \leq R \leq T)$ with straight lines. Theorem 1 below states that this piecewise linear function is $V_{T}$. Moreover, $V_{T}$ is concave (see the left frame of Figure 2).

We first set $\alpha=T$ and, for an arbitrary purchasing policy $\delta$, group purchases by their "replacement age." That is, for each $R=1, \ldots, T$, let $L_{R}$ be the purchase dates of all durables that are replaced at age $R$. Compute the weight $\lambda_{R}=\left(1-\beta^{R}\right) \sum_{t \in L_{R}} \beta^{t}$ and let $\lambda_{T+1}=1-\sum_{R=1}^{T} \lambda_{R}$. Roughly, the weight $\lambda_{R}$ corresponds to the fraction of purchased durables that are later replaced at age $R$. For example, if the policy is an $R$-fixed rule with $R<T+1$, then $L_{R}$ contains all the periods $t$ where $\delta_{t}=1$, so that $\lambda_{R}=1$ and $\lambda_{k}=0$ for all $k \neq R$. Let $(b, v)$ denote the budget and value of policy $\delta$. It turns out that:

$$
\left[\begin{array}{l}
b \\
v
\end{array}\right]=\sum_{R=1}^{T+1} \lambda_{R}\left[\begin{array}{l}
b_{T, R} \\
v_{T, R}
\end{array}\right] .
$$


Since the weights $\lambda_{R}$ are nonnegative and add up to 1 , the right-hand side is a convex combination of the two-dimensional vectors $\left\{\left(v_{T, R}, b_{T, R}\right)\right\}_{R=1}^{T+1}$. That is, the point $(b, v)$ must be in the convex hull of $\left\{\left(v_{T, R}, b_{T, R}\right)\right\}_{R=1}^{T+1}$, as depicted in Figure 2. Note that the upper frontier of this set coincides with the graph of the posited optimal value function $V_{T}$. Hence, $v \leq V_{T}(b)$. As we argue next, the upper bound $V_{T}(b)$ is attained by a particular type of flexible rule.

Suppose that $R$ is such that $b \in\left[b_{T, R+1}, b_{T, R}\right]$, and let $\delta^{*}$ be a policy that replaces durables at age $R$ or $R+1$ only. Such a policy is called an $R$-flexible rule. Its corresponding weights satisfy $\lambda_{k}^{*}=0$ for all $k \notin\{R, R+1\}$. By appropriately choosing the periods in which durables of age $R$ or age $R+1$ are replaced, we can also ensure that $b=\lambda_{R}^{*} b_{T, R}+\lambda_{R+1}^{*} b_{T, R+1}$ (as we explain later, this is always possible provided that $\beta$ is sufficiently large). Then, the value of $\delta^{*}$ is $\lambda_{R}^{*} v_{T, R}+\lambda_{R+1}^{*} v_{T, R+1}=V_{T}(b)$. That is, $\delta^{*}$ is optimal for the budget $b$.

For an arbitrary $\alpha$ now, let $b_{\alpha, R}$ and $v_{\alpha, R}$ denote the cost and the value of following the $R$-fixed rule when the endowed durable is of age $\alpha$. Then,

$$
\left[\begin{array}{c}
b_{\alpha, R} \\
v_{\alpha, R}
\end{array}\right]=\left[\begin{array}{c}
0 \\
X_{\alpha, R}
\end{array}\right]+\begin{gathered}
\beta^{R \ominus \alpha} \\
1-\beta^{R}
\end{gathered}\left[\begin{array}{c}
p_{0} \\
X_{0, R}
\end{array}\right] \quad \text { for all } R \leq T
$$

and $\left(b_{\alpha, T+1}, v_{\alpha, T+1}\right)=\left(0, X_{\alpha, T}\right)$. It is also convenient to define $b_{T+1, T+1}=p_{0}$ and $b_{0,1}=$ $\beta p_{0} /(1-\beta)$. Rules that replace goods more frequently require bigger budgets and have higher values. Hence, $b_{\alpha, R}>b_{\alpha, R+1}$ and $v_{\alpha, R}>v_{\alpha, R+1}$. Similar to $V_{T}$, the graph of the optimal value function $V_{\alpha}$ is obtained by joining the adjacent points $\left(b_{\alpha, R+1}, v_{\alpha, R+1}\right)$ and $\left(b_{\alpha, R}, v_{\alpha, R}\right)(1 \leq R \leq T)$ with straight lines (see Theorem 1 below). Figure 2 (right frame) simultaneously presents the optimal value functions $V_{1}, V_{2}$ and $V_{3}$ for the case when $T=3$.

Definition: Let $1 \leq R \leq T-1$ and $b \geq 0$. A policy $\delta$ is an $(R, b)$-flexible rule if it replaces durables only when they are of age $R$ or age $R+1$ and spends the budget $b$ exactly. If $\delta$ is an $(R, b)$-flexible rule, then for all $t, \delta_{t}=1$ implies that $\alpha_{t-1} \in\{R-1, R\}$.

Since an $(R, b)$-flexible rule sometimes replaces goods at age $R$, and sometimes at age $R+1$, it costs more than an $(R+1)$-fixed rule but less than an $R$-fixed rule. Hence, when the endowed good is of age $\alpha, b$ must be in the interval $\left[b_{\alpha, R+1}, b_{\alpha, R}\right]$. The $R$-fixed and the $(R+1)$-fixed rules are both special cases of the $(R, b)$-flexible rule for $b=b_{T, R}$ and $b=b_{T, R+1}$, respectively.

For $1 \leq \alpha, R \leq T$, let $A_{R}$ be the slope of $V_{\alpha}$ in the interval $\left(b_{\alpha, R+1}, b_{\alpha, R}\right)$ :

$$
A_{R}=\frac{v_{\alpha, R}-v_{\alpha, R+1}}{b_{\alpha, R}-b_{\alpha, R+1}}=\frac{1}{p_{0}}\left[X_{0, R}-\hat{x}_{R}\left[\frac{1-\beta^{R}}{1-\beta}\right]\right] \text {. }
$$


Note that $A_{R}$ is independent of $\alpha$, and it is easy to check that $A_{T}>A_{T-1}>\cdots>A_{1}>0$; therefore, $V_{\alpha}$ is indeed a concave function.

Theorem 1: Assume that

$$
\beta \geq \underline{\beta}(T)
$$

where $\underline{\beta}=1 / 2$ for $T=1$ and $\underline{\beta}$ is a (unique) positive root of $\underline{\beta}^{T}+\underline{\beta}^{T-1}=1$ for $T>1$. For each $\alpha=1, \ldots, T$, the optimal value function $V_{\alpha}$ is

$$
V_{\alpha}(b)=v_{\alpha, R+1}+A_{R}\left(b-b_{\alpha, R+1}\right), \quad b \in\left[b_{\alpha, R+1}, b_{\alpha, R}\right], \quad R=T, \ldots, 1,
$$

and for any budget $b \geq 0$, the corresponding optimal purchasing policy is an $(R, b)$-flexible rule, where $R$ is such that $b \in\left[b_{\alpha, R+1}, b_{\alpha, R}\right]$ (when $b=b_{\alpha, R}$, this policy coincides with the $R$-fixed rule). More precisely, the optimal purchasing policy is given by

$$
\delta_{\alpha}^{*}(b)= \begin{cases}0 & \text { for } b<b_{\alpha+1, \alpha+1} \\ \{0,1\} & \text { for } b_{\alpha+1, \alpha+1} \leq b \leq b_{\alpha-1, \alpha} \\ 1 & \text { for } b>b_{\alpha-1, \alpha}\end{cases}
$$

Proof: See Appendix.

Assumption (8) plays the same role as $\beta \geq \frac{1}{2}$ in Section 3. This assumption guarantees that it is feasible to spend any budget $b \in\left[b_{\alpha, R+1}, b_{\alpha, R}\right]$ by following an $(R, b)$-flexible rule, and this property is essential for (9) to represent the optimal value function. To understand why, consider an $R$-flexible consumer in state $\alpha=R$ when she is about to replace the durable. Focus, in particular, on the budget $b$ in the indifference region for $\delta_{R}^{*}(b)$ in $(10)$,

$$
b \in\left[b_{R+1, R+1}, b_{R-1, R}\right]=\left[\frac{p_{0}}{1-\beta^{R+1}}, \frac{\beta p_{0}}{1-\beta^{R}}\right] .
$$

When the budget falls in the indifference region, the optimal decision rule $\delta_{R}^{*}(b)$ allows the consumer to either keep or replace the durable. If she keeps the good $\alpha=R$ now, she would replace it for sure at age $R+1$ and will, therefore, revisit the state $\alpha=R$ in the next $R+1$ periods with a durable budget $b^{\prime}=\frac{1}{\beta^{R+1}}\left(b-\beta p_{0}\right)$. Similarly, if the consumer replaces the good now (at age $R$ ), she will revisit the state $\alpha=R$ in the next $R$ periods with a budget $b^{\prime \prime}=\frac{1}{\beta^{R}}\left(b-p_{0}\right)$. Assumption (8) guarantees that for any $b \in\left[b_{R, R+1}, b_{R, R}\right]$, the subsequent budgets $b^{\prime}, b^{\prime \prime}$ will continue to fall into the same $R$-flexible rule budget range $\left[b_{R, R+1}, b_{R, R}\right]$. Hence, it is feasible to follow the $R$-flexible rule from any initial state $(\alpha, b)$, with $b \in\left[b_{\alpha, R+1}, b_{\alpha, R}\right]$.

Now, suppose that assumption (8) does not hold. Then, $b_{R-1, R}<b_{R+1, R+1}$, at least for 
$R=T-1$ and maybe for some $R<T-1$, as well. We take a budget $\tilde{b} \in\left[b_{R-1, R}, b_{R+1, R+1}\right] \subset$ $\left[b_{R, R+1}, b_{R, R}\right]$ and calculate the future budgets $b^{\prime}, b^{\prime \prime}$ at the next revisit point $\alpha=R$. Simple algebra shows that

$$
b^{\prime}=\frac{1}{\beta^{R+1}}\left(\tilde{b}-\beta p_{0}\right)>b_{R, R}, b^{\prime \prime}=\frac{1}{\beta^{R}}\left(\tilde{b}-p_{0}\right)<b_{R, R+1}
$$

We can see that the consumer with budget $\tilde{b}$ cannot follow the prescribed $R$-flexible rule in the future, because both $\delta_{R}=0$ and $\delta_{R}=1$ decisions take her outside of the $R$-flexible rule budget set the very next time she revisits the state $\alpha=R$. In other words, when (8) is violated, there is no $R$-flexible rule that can spend a budget such as $\tilde{b}$, and the optimal value function is no longer given by (9).

Assumption (8) puts a lower bound on the discount rate for one period. For a fixed annual interest rate $\rho$, the discount rate $\beta$ corresponds to a period length of $l=\frac{1}{\rho} \ln \frac{1}{\beta}$ years and the useful lifetime of the durable of $T \cdot l$ years. Accordingly, a lower bound on $\beta$ is equivalent to an upper bound $\bar{L}_{T}=\frac{T}{\rho} \ln \frac{1}{\underline{\beta}(T)}$ on the useful lifetime. For $\rho=0.02$, a typical value used in most of the literature on consumer intertemporal choice, $\bar{L}_{T}$ is between 34.6 and 48.1 years, depending on $T$. In most high-innovation markets, where our model is plausibly applicable, durables become completely obsolete long before age 35. Hence, assumption (8) does not seem restrictive for empirically relevant parameter values.

We now establish the optimality of no-delay policies within the broader class of all feasible durable replacement rules. The general durable replacement policy is a sequence of pairs $\left\{\tau_{k}^{\prime}, d_{k}\right\}$, where $\tau_{k}^{\prime}$ is the model number and $d_{k} \in[0,1)$ is the "delay" of the $k$-th purchase, so the time of the $k$-th purchase is $\tau_{k}^{\prime}+d_{k}$. The following theorem states that it is optimal to set $d_{k}=0$ for all $k$.

Theorem 2: For each $\alpha=1, \ldots, T$, the optimal value function $V_{\alpha}(b)$ is that given by (9) in Theorem 1. For any budget $b \geq 0$, the corresponding optimal purchasing policy $\left\{\left(\tau_{k}^{\prime}, d_{k}\right)\right\}_{k \geq 1}$ has $d_{k}=0$ for all $k$ and is an $(R, b)$-flexible rule, where $R$ is such that $b \in$ $\left[b_{\alpha, R+1}, b_{\alpha, R}\right]$.

Proof: See Appendix.

We now present a sketch of the proof for Theorem 2. Suppose that an agent replaces her durable of age $R$ with delay in the current period. Consider the costs and benefits from this delay, given that there are no delays in the future. If the consumer increases the delay of the replacement by $d t$, she forgoes service flow $\left(x_{0}-x_{R}\right) d t$ but increases her wealth by $p_{0} \rho d t$, the amount of interest saved by purchasing the good later. Theorem 1 implies that the marginal utility of wealth for someone who follows an $R$-flexible rule is less than or equal 
to $A_{R}$. Simple algebra shows that

$$
\rho p_{0} A_{R} \leq x_{0}-x_{R}
$$

so the gain from delay is less than the corresponding loss of service.

The proof of the theorem generalizes this argument by showing that similar reasoning applies when more than one durable purchase is delayed. An arbitrary policy with delays can be modified recursively by eliminating one delay at a time while maintaining the same budget and improving its value.

Physical DePRECiATion: The results of Theorems 1 and 2 generalize in a straightforward fashion if we allow deterministic physical depreciation within a period. Suppose that the service flow from the good of age $\alpha \geq 1$ equals $x_{\alpha} \cdot \Delta(s)$, where $s \in[0,1]$ is the time elapsed since the beginning of the current period, and $\Delta(\cdot)$ is a decreasing function that describes physical depreciation during a period. The amount of physical depreciation depends on the time elapsed since the date of purchase. To capture this, assume that for $\alpha=0$, the service flow from the durable purchased at time $d \leq s$ equals $x_{0} \cdot \Delta(s-d)$. Let $\Delta(0)=1$ and $\Delta(1)>\max _{\alpha}\left(\frac{x_{\alpha+1}}{x_{\alpha}}\right)$ to guarantee that the service flow from the durable is non-increasing. As before, define the average service flow per period,

$$
\hat{x}_{\alpha}=x_{\alpha} \int_{0}^{1} e^{-\rho s} \Delta(s) d s
$$

By construction, $\hat{x}_{\alpha}$ is a non-increasing sequence, so Theorem 1 holds. To see if Theorem 2 holds, consider the costs and benefits of a single delay. The service flow forgone by delaying a durable purchase by $d t$ now depends on $d$ and equals $\left[x_{0} \cdot \Delta(0)-x_{R} \cdot \Delta(d)\right] d t$. That is, since the current durable experiences physical depreciation, the forgone service flow rises with delay and is always greater than $\left(x_{0}-x_{R}\right) d t$. Hence,

$$
\rho p_{0} A_{R} \leq x_{0}-x_{R}<x_{0}-x_{R} \cdot \Delta(d)
$$

and we can see that physical depreciation strengthens the incentive to replace durables without delay. Thus, Theorem 2 extends to the case with physical depreciation.

\subsection{Optimal budget allocation}

We now solve the budget allocation problem (4). Consider an agent with wealth $w$ and a durable of age $\alpha$. As we argued before, if the agent allocates a budget $b$ for durable consumption, then she optimally spends $c=(1-\beta)(w-b)$ per period on non-durables. 
The agent should pick $b$ so as to equate the marginal utilities of durable ${ }^{12}$ and non-durable consumption:

$$
V_{\alpha}^{\prime}(b)=\hat{u}^{\prime}((1-\beta)(w-b)) .
$$

Figure 3 depicts the marginal utility of durable consumption (the falling step-function because $V_{\alpha}$ is a concave piecewise linear function) and the marginal utility of consumption as functions of $b$ (for given values of $\alpha$ and $w$ ).

In the left panel of Figure $3, \hat{u}^{\prime}((1-\beta)(w-b))$ crosses $V_{\alpha}^{\prime}(b)$ at a point of discontinuity. In the figure, the optimal durable budget equals $b_{\alpha, R}$ and the corresponding durable purchasing policy is the $R$-fixed rule. Now, decrease $w$ by a small amount. The graph of $\hat{u}^{\prime}((1-\beta)(w-b))$ will shift to the left, but it will still cross $V_{\alpha}^{\prime}$ at $b=b_{\alpha, R}$. In other words, there is an interval of wealths $w$ for which it is optimal to follow the $R$-fixed rule from the state $(\alpha, w)$. If we further decrease $w, \hat{u}^{\prime}$ will eventually cross $V_{\alpha}^{\prime}$ at a point where $V_{\alpha}^{\prime}$ is flat and equal to $A_{R}$. In this case, it is optimal to choose a budget corresponding to an $R$-flexible rule and pick the non-durable budget $c_{R}$, where $\hat{u}^{\prime}\left(c_{R}\right)=A_{R}$. Hence, there is also an interval of wealths $w$ for which it is optimal to follow the $R$-flexible rule and spend $c_{R}$ on non-durables every period. For that range of wealths, the optimal non-durable budget remains constant and variations in wealth affect the durable consumption path only (higher wealth affords replacing durables at age $R$ more frequently, while lower wealth requires replacing durables at age $R+1$ more often). In contrast, when a fixed rule is optimal, higher wealth leads to a higher level of non-durable consumption.

For a fixed $\alpha$, if $w$ varies continuously from infinity to zero, the intersection of $\hat{u}^{\prime}((1-$ $\beta)(w-b))$ with $V_{\alpha}^{\prime}(b)$ in Figure 3 moves monotonically to the left and maps out the optimal decision rule (as a function of $w$ ). The wealthiest consumers use a 1-fixed rule. Next comes a group of consumers that follow a 1-flexible rule, and then a group that follows the 2-fixed rule, and so on. The intervals of wealth where agents follow fixed rules are interlaced with the intervals of wealth where they follow flexible rules. The bounds of these intervals can be computed explicitly. Let

$$
w_{\alpha, R}(c)=\frac{c}{1-\beta}+b_{\alpha, R}
$$

be the wealth required to follow the $R$-fixed rule and spend a constant non-durable budget $c$ per period when the initial durable is of age $\alpha$. The wealthiest agent that follows the $R$-flexible rule replaces her durable every $R$ periods and consumes $c_{R}$. Hence, her wealth is $w_{\alpha, R}\left(c_{R}\right)$. The poorest agent that follows the $(R-1)$-flexible rule also replaces her durable

\footnotetext{
${ }^{12}$ The piecewise linear function $V_{\alpha}$ is not differentiable at the budgets $b_{\alpha, R}$, but since $V_{\alpha}$ is concave, it has a well defined interval of subgradients at $b_{\alpha, R}$, which, with abuse of notation, we still denote by $V_{\alpha}^{\prime}\left(b_{\alpha, R}\right) \in\left[A_{R}, A_{R-1}\right]$.
} 
every $R$ periods but consumes $c_{R-1}>c_{R}$, so that her wealth is $w_{\alpha, R}\left(c_{R-1}\right)>w_{\alpha, R}\left(c_{R}\right)$. In between, there are consumers with wealth $w \in\left[w_{\alpha, R}\left(c_{R}\right), w_{\alpha, R}\left(c_{R-1}\right)\right]$ that follow the $R$-fixed rule. Each one spends the same durable budget $b_{\alpha, R}$ and the non-durable budget per period

$$
c_{\alpha, R}(w)=(1-\beta)\left(w-b_{\alpha, R}\right) .
$$

A consumer with more wealth than $w_{1,1}\left(c_{1}\right)=\left(c_{1}+p_{0}\right) /(1-\beta)$ will replace her durable every period and spend more than $c_{1}$ per period on non-durables. We will assume that $\bar{y} / \rho \geq w_{1,1}\left(c_{1}\right)$, and define $\bar{w}=\bar{y} / \rho$ and $c_{0}=(1-\beta) \bar{w}-p_{0}$. Similarly, a consumer with less wealth than $c_{T} /(1-\beta)$ will spend all her wealth on non-durable consumption. We will assume that $\underline{y} / \rho \leq c_{T} /(1-\beta)$, and define $\underline{w}=\underline{y} / \rho$ and $c_{T+1}=(1-\beta) \underline{w}$.

We can also express the optimal purchasing policy (10), stated in Theorem 1 , as a function of $w$ (and, with abuse of notation, denote this function by the same symbol $\delta_{\alpha}^{*}$ ). The following theorem states these results formally.

Theorem 3: Let $c_{0}=(1-\beta) \bar{w}-p_{0}, c_{T+1}=(1-\beta) \underline{w}$, and for each $R=1, \ldots, T$, let $c_{R}$ be such that $\hat{u}^{\prime}\left(c_{R}\right)=A_{R}$. Denote by $b_{\alpha}^{*}(w)$ the optimal solution of problem (4). Then, for $\alpha=1, \ldots, T, b_{\alpha}^{*}(w)=w-c_{\alpha}^{*}(w) /(1-\beta)$, where

$$
c_{\alpha}^{*}(w)= \begin{cases}c_{\alpha, R}(w) & \text { for } w \in\left[w_{\alpha, R}\left(c_{R}\right), w_{\alpha, R}\left(c_{R-1}\right)\right], \quad R=T+1, \ldots, 1 \\ c_{R} & \text { for } w \in\left[w_{\alpha, R+1}\left(c_{R}\right), w_{\alpha, R}\left(c_{R}\right)\right], \quad R=T, \ldots, 1\end{cases}
$$

and

$$
\delta_{\alpha}^{*}(w)= \begin{cases}0 & \text { for } w<w_{\alpha+1, \alpha+1}\left(c_{\alpha}\right) \\ \{0,1\} & \text { for } w_{\alpha+1, \alpha+1}\left(c_{\alpha}\right) \leq w \leq w_{\alpha-1, \alpha}\left(c_{\alpha}\right) \\ 1 & \text { for } w>w_{\alpha-1, \alpha}\left(c_{\alpha}\right)\end{cases}
$$

Proof: See Appendix.

Over time, a consumer that follows an $R$-fixed rule has a constant holding time $R$ and revisits the same points in the state space $(\alpha, w)$ every $R$ periods. Her wealth trajectory is cyclical. While the consumer keeps the current good, both $\alpha$ and $w$ increase, as the consumer "saves" for the next purchase. When the consumer buys a new durable, both $\alpha$ and $w$ go down, and the holding cycle starts again.

The time path for wealth of a consumer that follows an $R$-flexible rule is more erratic. Usually, her wealth trajectory is not cyclical: each time the durable is of age $R$, she has a different wealth level (recall the discussion in Section 3). Thus, the agent will switch replacement frequencies in a seemingly erratic pattern, as each time that she revisits the state $\alpha=R$, her wealth level $w$ falls into a different place on the interval $\left[w_{R+1, R}\left(c_{R}\right), w_{R, R}\left(c_{R}\right)\right]$. 


\subsection{Secondary markets}

Many durable goods have active resale markets, and it is informative to investigate how opening the used-goods market affects our main results. We start with the simplest case $T=1$. The used market might potentially play a role in allocating used goods with lower service flows to less-affluent consumers. Accordingly, for the purposes of this section, we assume that the durable experiences physical depreciation, as in (12). The durable of age $s \in[0,1]$ provides instantaneous utility $x_{0} \Delta(s)$. Depending on the range of $\Delta(s)$, there may be a continuum of used goods with distinct instantaneous utilities in the range $\left[0, x_{0}\right]$. We assume that there are no trading frictions, allowing consumers to realize any positive gains from trade in the used market.

Before stating results formally, we develop some relevant intuitions. When $T=1$, there are just two classes of consumers: the 1-fixed rule class and the 1-flexible rule class. Think in terms of an efficient allocation of used goods across consumers. It is not efficient to allocate used goods to 1-fixed rule consumers because they want to further increase their durable consumption, but are constrained by the maximum attainable durable utility $\frac{x_{0}}{\rho}$. This leaves the 1-flexible rule consumers as the only potential used-good buyers. Trades within the flexible rule class are a wash because all members of this group have identical marginal utilities of wealth, $\hat{u}\left(c_{1}\right)=A_{1}$, despite having different $w$. Alternatively, the 1flexible class could be induced to buy used goods from the 1-fixed class at sufficiently low prices. However, any price acceptable to the buyers will be too low to be acceptable to the sellers. Hence, there are no gains from trade, and the used market is redundant. Formally, we have

Proposition 2 Let $T=1$, and let the service flow from the durable of age $s \in[0,1]$ be given by $x_{0} \Delta(s)$. Then, the gains from trade in the used market are, at most, zero.

Proof: See Appendix.

Proposition 2 shows that the used market is redundant for $T=1$, even if assumed physical depreciation generates a continuum of distinct used goods. In the special case, there is no resale because the 1-fixed rule consumers cannot be induced to sell their durables until age 1 , and everyone else has zero gains from trade.

The main argument in Proposition 2 generalizes to the case when $T>1$. It is inefficient for 1-fixed rule consumers to sell used goods until they reach age 1 because doing so will reduce their utility from the durables and make it necessary to spend even more on nondurables that have low marginal utility $\hat{u}^{\prime}(c) \leq A_{1}$ to begin with. Hence, successive cohorts of 1-period-old goods will come on the market at dates $\tau \in \mathbf{N}$. Accordingly, the potential buyers of these 1-period-old used goods have an incentive to time their own resale decisions 
with dates $\tau$ and offer their old units for sale at the same time. By induction, we expect that this mechanism will generate supply spikes for multiple older vintages of used goods precisely at dates $\tau$ when 1-fixed rule consumers choose to trade. Beyond this, an analytical characterization of the used market equilibrium for $T>1$ becomes difficult because of the combinatorics associated with a large number of distinct replacement rules that can potentially be part of equilibrium. Nevertheless, the above argument demonstrates why the demand for new goods may remain periodic even when the used markets have active trade.

\section{$5 \quad$ Aggregate Implications}

The suitability of our model for analyzing aggregate phenomena outside of a narrow sector depends on the assumptions that we are willing to make on the innovation process underlying the introduction of better products. If there are numerous durable-good categories and innovations are uncorrelated across categories, sectoral demand fluctuations will wash out in the aggregate. The above description of innovation is plausible and analytically convenient, but it is not complete: historians studying creative destruction point out that drastic innovations are important drivers of progress, and that some of these innovations are radical enough to make an aggregate impact (e.g., Mokyr, 1990, a, b). Accordingly, another prominent branch of the creative destruction literature (see, in particular, Helpman and Trajtenberg, 1998; Laitner and Stolyarov, 2004; and Jovanovic and Rousseau, 2005) stresses the importance of general purpose technologies (GPT) that are so pervasive as to affect the entire economy. Generally, any technology with wide applicability could induce nearly simultaneous innovations across multiple sectors and make a model like ours potentially useful for studying demand at higher levels of aggregation. ${ }^{13}$ Battery technology is one recent example. Power supply has been a constraint in adding features to portable devices across multiple categories; it also has been a major factor affecting development of new propulsion technologies in transportation. Hence, a substantial improvement in battery energy density might simultaneously bring about feature-rich smart phones, lightweight power tools and long-range electric cars.

\footnotetext{
${ }^{13}$ When technological change is assumed to have an aggregate impact, periodic innovations can induce movements in the equilibrium interest rate. Some features of the optimal policy will be affected - for instance, non-durable consumption will become time-varying; however, we conjecture that the non-standard features of consumer behavior emphasized in the rest of this section will still be present in general equilibrium. This is because certain classes of consumers (e.g., the upper tail of the wealth distribution) will replace durables without delay and will follow fixed rules even when the interest rate is not constant. The full general equilibrium characterization is outside of the scope of this paper; nevertheless, some results from partial equilibrium analysis can be relevant in general equilibrium, as well.
} 


\subsection{Consumption response to a change in wealth}

Durable and non-durable consumption show different responses to changes in wealth. Fixedrule consumers have a fixed durable budget and a positive marginal propensity to consume non-durables (see Figure 3). Therefore, if any such consumer receives windfall income, she will spend it all on non-durable consumption. By contrast, flexible-rule consumers have a zero marginal propensity to consume non-durables and a variable durable budget. ${ }^{14}$ They will save all of their windfall to make future durable purchases and will not change their nondurable consumption. Leahy and Zeira (2005) call this the "insulation effect": the windfall is entirely absorbed by changes in durable purchase timing, and non-durable consumption is, thus, insulated from the wealth shock.

The insulation effect highlights durable purchase timing as a potentially important propagation channel for income and wealth shocks. Interestingly, comparisons between our model and that of Leahy and Zeira (2005) reveal that the underlying mechanisms for consumption insulation are very different. In both models, the insulation effect arises because the agent's marginal utility of wealth is independent of purchase timing ${ }^{15}$ (for $R$-flexible-rule consumers it is constant and equal to $A_{R}$, for example). In our model, the insulation effect is a consequence of restricted choices of purchase timing: flexible rules arise precisely because it is optimal to adjust holding periods for the durable only in discrete increments. In Leahy and Zeira, by contrast, the insulation effect obtains mostly due to unrestricted choice of purchase dates, but restricted choice of the holding period for the durable.

\subsection{Volatility of durable consumption}

It is well known that aggregate durable consumption is more volatile than aggregate nondurable consumption. Our analysis explains why the demand for durables may exhibit strong fluctuations even in the absence of income or wealth shocks. In the deterministic version of the model, the time series volatility of durable and non-durable expenditure are at opposite extremes. That is, the standard deviation of the growth rate of durable expenditure is large because durable purchases exhibit spikes at discrete dates. Since non-durable consumption is constant, the standard deviation of its growth rate is zero.

In reality, consumers may experience idiosyncratic shocks to their permanent income or to the service flow from their current durable. Consumers subject to idiosyncratic shocks

\footnotetext{
${ }^{14}$ The obsolescence process that controls $x_{\alpha}$ determines the boundaries of wealth intervals corresponding to the various fixed-rule and flexible-rule groups and, thus, selects where and when the consumers spend their windfall, and the size of the wealth shock determines how much they spend.

${ }^{15}$ The additively separable utility function and the interest rate at the steady-state level are necessary conditions for this property in both models.
} 
cannot perfectly plan ahead all their durable purchases, and they will make some purchases in the middle of the cycle. We expect that large idiosyncratic shocks will moderate the volatility of durable expenditure, while making the volatility of non-durable consumption positive. The distinct implication of our model is that higher income variance (e.g., Haider, 2001) can have opposite effects on the volatility of durables and non-durables: it can lower the volatility of durable demand, while increasing the variance of non-durable consumption.

\section{Random Period Length}

In this section, we solve the durable replacement problem with random arrival times, focusing on the "diffusion curve" (the number of users of the new model as a function of time since its introduction). In our basic framework with certainty, the consumers can perfectly predict when new models will arrive at the market. The diffusion of every new model of the durable is, therefore, instantaneous (L-shaped): all consumers who purchase model $\tau$ do so simultaneously at time $\tau$. By contrast, in a model with Poisson arrivals, the hazard rate of model introduction stays constant, and the expected service flow of a new durable is independent of the time of purchase. Therefore, consumers have no incentive to time their purchases near the model introduction dates. The diffusion of the new model will happen with inertia, with the shape of the diffusion curve determined by the distribution of wealth.

We adopt a framework that combines these two extreme cases. We assume that the development times of new models are i. i. d. random variables distributed on $[S, \infty)$, where $S \geq 0$ is the minimum gestation period. If $\tau$ is the time it takes to develop and introduce a new model into the market, then $\tau-S$ has an exponential distribution with parameter $\lambda$. Thus, the average arrival time is $S+1 / \lambda$. Note that our deterministic model is the limit case when $S=1$ and $\lambda \rightarrow \infty$, and that the pure Poisson arrival model corresponds to the case when $S=0$ and $\lambda>0$. Our goal is to demonstrate that the diffusion curve features an adoption plateau: there is a group of early adopters who purchase the new model right away, followed by no purchases at all for a while (plateau), followed by gradual purchases by late adopters.

For tractability, we assume that there is only one good, the durable, and that each agent has a lifetime budget $b$ to spend on durables. ${ }^{16}$ This model focuses exclusively on the

\footnotetext{
${ }^{16}$ Because we assumed that durable budgets are exogenously determined, there is not an exact correspondence between the durable purchasing policy and the solution to an optimal consumption problem with two goods. In a dynamic optimization problem with two goods, the agents will optimally reallocate their budgets between durables and non-durables when new information becomes available. For example, if the design cycle has been unexpectedly long, an agent may want to start using part of his durable budget to increase her non-durable consumption.
} 
intertemporal trade-off of purchasing the durable at different points in time, and it excludes the possibility of an ongoing substitution between the durable and other goods. Since we are primarily interested in the timing of durable purchases, this simplified specification seems appropriate.

In many durable-goods markets, the quality-adjusted price for the durable falls over time because of manufacturing efficiency improvements. Thus, we now also let the price of the durable fall exponentially over time: $p_{t}=p_{0} e^{-\gamma t}$. With falling prices, it may become attractive to buy a durable with delay: though its expected service flow decreases, the durable also becomes cheaper.

The state variables for the consumer are $\alpha$ - the technological age of the endowed durable; $\bar{b}_{t}=b_{t} / p_{t}$ - the "purchasing power" of the consumer; and $s$ - the time since the last arrival of a new model (i.e., the age of the current model). The law of motion for the purchasing power is

$$
\bar{b}_{t+\Delta t}=\left(\bar{b}_{t}-\delta_{t}\right) e^{(r+\gamma) \Delta t}
$$

where, as before, $\delta_{t}=1$ if a new durable is purchased at date $t$ and $\delta_{t}=0$ otherwise.

Let $V_{\alpha}(\bar{b}, s)$ be a consumer's total discounted value of holding a purchasing power $\bar{b}$ and a durable of technological age $\alpha$ at the moment when a time $s$ has elapsed since the last arrival of a new model. We restrict attention to the case $T=1$, so $\alpha \in\{0,1\}$. This captures the main insights from the extended model without making the proofs excessively complicated.

When the new model reaches age $S$, innovations start arriving at a constant Poisson rate $\lambda$, and $s$ becomes uninformative about the time of the next arrival. Therefore, the value function ceases to depend on $s$ :

$$
V_{\alpha}(\bar{b}, s)=V_{\alpha}(\bar{b}, S), s \geq S
$$

$V_{0}(\bar{b}, S)$ is the value of the service flow $x_{0}$ until the next Poisson event and the continuation value of an old model afterwards:

$$
\begin{aligned}
V_{0}(\bar{b}, S) & =\int_{0}^{\infty} \lambda e^{-\lambda t}\left[\int_{0}^{t} e^{-\rho \tau} x_{0} d \tau+e^{-\rho t} V_{1}\left(\bar{b} e^{(r+\gamma) t}, 0\right)\right] d t \\
& =\underset{\lambda+\rho}{x_{0}}+\int_{0}^{\infty} \lambda e^{-(\lambda+\rho) t} V_{1}\left(\bar{b} e^{(r+\gamma) t}, 0\right) d t .
\end{aligned}
$$

For $s<S$,

$$
V_{0}(\bar{b}, s)=\int_{0}^{S-s} x_{0} e^{-\rho \tau} d \tau+e^{-\rho(S-s)} V_{0}\left(\bar{b} e^{(r+\gamma)(S-s)}, S\right) .
$$

Replacement DeCision: Every time a new model arrives, all existing durables depreci- 
ate. An agent with purchasing power $\bar{b}$ chooses the delay $d(\bar{b})$ in purchasing the new model. It is convenient to distinguish between two cases $-d<S$ and $d>S$ - and to separate the optimization problems over these intervals. Call the corresponding value functions $V_{1}^{L}(\bar{b})$ and $V_{1}^{R}(\bar{b})$. Both of these value functions are measured at the point where $s=0$. Then,

$$
V_{1}(\bar{b}, 0)=\max \left\{V_{1}^{L}(\bar{b}), V_{1}^{R}(\bar{b})\right\}
$$

where

$$
V_{1}^{L}(\bar{b})=\max _{0 \leq d \leq S}\left(\frac{x_{1}\left(1-e^{-\rho d}\right)}{\rho}+e^{-\rho d} V_{0}\left(\bar{b} e^{(r+\gamma) d}-1, d\right)\right)
$$

and

$$
\begin{aligned}
V_{1}^{R}(\bar{b})=\max _{d \geq S} \int_{0}^{S} x_{1} e^{-\rho \tau} d \tau & +e^{-\rho S} \int_{0}^{d-S} \lambda e^{-\lambda t}\left[\int_{0}^{t} x_{1} e^{-\rho \tau} d \tau+e^{-\rho t} V_{1}\left(\bar{b} e^{(r+\gamma)(t+S)}, 0\right)\right] d t \\
& +e^{-\lambda(d-S)}\left(\int_{S}^{d} x_{1} e^{-\rho \tau} d \tau+e^{-\rho d} V_{0}\left(\bar{b} e^{(r+\gamma) d}-1, S\right)\right)
\end{aligned}
$$

The first term in (16) is the value of holding the depreciated good until the new model reaches age $S$; the second term is the expected value of holding the depreciated good between $S$ and $d$; and the third term is the expected value of the replacement at $d$. (Note that when a new good is bought at the time when $s>S$, the corresponding continuation value of a new durable is as if it were of age $S$.) The following proposition characterizes the optimal delay.

Proposition 3: Let $\rho=r+\gamma$ and let $S>0$. Then, there is an interval $[\underline{S}, \bar{S}]$ with $0 \leq$ $\underline{S}<S<\bar{S}$ such that no durable purchases are made in the interval $[\underline{S}, \bar{S}]$ after new model arrivals. That is,

$$
d(\bar{b}) \leq \underline{S} \text { or } d(\bar{b}) \geq \bar{S} \text { for all } \bar{b} \geq 0 .
$$

Moreover, all consumers who can afford the new model either purchase it right away (when $s=0)$ or after date $S$ :

$$
d(\bar{b})=0 \text { or } d(\bar{b}) \geq \bar{S} \text { for all } \bar{b} \geq 1 .
$$

Furthermore, if $S=0$, then $\underline{S}=\bar{S}=0$.

Proof: See Appendix.

Proposition 3 illustrates how predictability of innovations affects the adoption timing. When $S=0$ and the hazard rate of innovation is constant (i.e. arrival is unpredictable), demand for the durable stays positive from the time of innovation until the time when all consumers have purchased the current model. By contrast, when $S>0$, demand for the durable falls to zero as the date of possible new arrivals (that is, date $S$ ) draws sufficiently 
close and stays at zero for some time after date $S .{ }^{17}$ In other words, early adopters act before time $\underline{S}$; no one adopts between $\underline{S}$ and $\bar{S}$; and late adopters postpone action until after time $\bar{S}$. Thus, the no-purchase interval represents the flat part of the diffusion curve where the number of adopters stays constant over time. Figure 4 illustrates a typical diffusion curve that arises in this case.

We investigate numerically whether the adoption plateaus are quantitatively important, especially when the price of the durable is falling. For the simulations, we choose parameter values that we think are representative of markets for high-tech products. If $x_{0}$ and $x_{1}$ are the service flows of the durables in a detrended model, then the corresponding absolute service flows are $z_{\tau}=e^{x_{\tau}}, \tau=0,1$. Hence, $z_{0} / z_{1}=e^{\left(x_{0}-x_{1}\right)}$ is the relative advantage of a new model. We set $x_{0}=0.4$ and $x_{1}=0$, which corresponds to a new model providing 50 percent more service than old models. We perform simulations for two average design cycle lengths: $L=S+1 / \lambda=\{3,5\}$ years. We set parameters $\rho=r=0.04$ and experiment with different values of $\gamma, S$ and $\lambda$.

Figure 5 shows our results. In each panel, purchase delay is on the vertical axis and $\lambda$ is on the horizontal axis. The average introduction time $L$ is three years for the left panels and five years for the right panels, while $\gamma=0.05$ for the top panels and $\gamma=0.12$ for the bottom panels. Since, in each panel, the average introduction time is kept constant, as $\lambda$ varies, $S$ needs to be adjusted accordingly. Let $S(\lambda)=L-1 / \lambda$. This function is plotted as a thin solid line in each panel. The thick lines show the ends of the no-purchase interval, $\underline{S}(\lambda)$ and $\bar{S}(\lambda)$. In all cases, $\underline{S}(\lambda) \leq S(\lambda) \leq \bar{S}(\lambda)$. All three lines cross at $S=0$, which corresponds to the case of pure Poisson arrival times.

Note that when $\gamma=0.05, \underline{S}=0$. When the average introduction time is $L=3$ (topleft panel), note, also, that $\bar{S}>L$ for $\lambda \geq 0.4$. For those parameters, the time between new arrivals will often be shorter than $\bar{S}$. That is, the consumers who wanted to delay the purchase beyond $\bar{S}$ are frequently 'surprised' by a new arrival before the time when they were prepared to buy a new model. Obviously, when this happens, the consumers begin the new cycle with higher purchasing power; and those with a high enough $\bar{b}$ will buy the new model right away. That is, for some consumers, the surprise provokes an earlier purchase than what was 'scheduled.' This means that for a given realization of model arrival times, the majority of the purchases will be perfectly synchronized with the arrival dates, as in the deterministic setting. When $\gamma=0.12, \underline{S}>0$ for sufficiently high $\lambda$. In this case, the initial purchase spike, produced by those consumers with $d(\bar{b})=0$, is followed by a period

\footnotetext{
${ }^{17}$ The latter result mirrors our Theorem 2 , where we showed that new models are always purchased without delay for $r=\rho$ and $\gamma=0$. By continuity, Proposition 3 should also hold when $r=\rho$ and $\gamma>0$ is sufficiently small.
} 
of positive demand (until $\underline{S}$ ). Afterwards, demand drops to zero, and purchases resume only if the innovation cycle turns out to be sufficiently long.

\section{Conclusion}

We develop a model of durable goods that highlights the difference between obsolescence and physical wear and tear. The basic model is simple and can be solved analytically. We identify predictable obsolescence as a distinct source of demand fluctuations and explain how it affects technology adoption decisions. The key implications of the basic model carry over to the case with stochastic obsolescence and falling relative price of the durable. The stochastic model produces a diffusion curve with an adoption plateau, which is in contrast to the standard, S-shaped, logistical diffusion curve.

The model explains simultaneous adoption of new durables without relying on network effects or externalities. This result provides a new perspective on policies that are designed to encourage mass technology adoption (e.g., a subsidy for digital TV). What is more, our analysis explains why durable and non-durable consumption might respond to wealth and income shocks in non-standard ways. Specifically, in our model, larger income shocks reduce the volatility of durable demand but increase the volatility of non-durable demand. Also, the possibility of shifting durable purchase timing in response to changes in wealth leads to an insulation effect for non-durable consumption. The above results may inform empirical studies of demand in markets in which obsolescence cycles are important. 


\section{References}

[1] Balcer, Yves; Lippman, Steven A., "Technological Expectations and Adoption of Improved Technology", Journal of Economic Theory. December 1984; 34(2): 292-318.

[2] Bannerjee, A., 1992. "A simple model of herd behavior". Quarterly Journal of Economics 107, 797-817.

[3] Boucekkine, Raouf, David de la Croix and Omar Licandro, "Vintage Capital Growth Theory: Three Breakthroughs", Barcelona GSE Working Paper Series Working Paper 565 , June 2011.

[4] Comin, Diego, Bart Hobijn and Emilie Rovito, "Five Facts You Need To Know About Technology Diffusion", National Bureau of Economic Research working paper 11928, January 2006.

[5] Comin, Diego, and Bart Hobijn. "An Exploration of Technology Diffusion." American Economic Review 100, no. 5 (December 2010): 2031-59.

[6] Cooper, Russell; Haltiwanger, John, (1993a) "The Aggregate Implications of Machine Replacement: Theory and Evidence". American Economic Review. June 1993; 83(3): 360-82.

[7] Cooper, Russell; Haltiwanger, John, (1993b) "Automobiles and the National Industrial Recovery Act: Evidence on Industry Complementarities". Quarterly Journal of Economics. November 1993; 108(4): 1043-71.

[8] Dranove, David and Gandal, Neil, "The DVD-vs.-DIVX Standard War: Empirical Evidence of Network Effects and Preannouncement Effects", Journal of Economics \& Management Strategy, Volume 12, Number 3, Fall 2003, 363-386

[9] Farrell, Joseph; Saloner, Garth, "Standardization, Compatibility, and Innovation" RAND Journal of Economics. Spring 1985; 16(1): 70-83.

[10] Fishman, A. and R. Rob, "Product Innovation by a Durable-Good Monopoly", RAND Journal of Economics 31(2), 237-52, 2000.

[11] Francois, Patrick ; Lloyd-Ellis, Huw "Animal Spirits through Creative Destruction", The American Economic Review, Vol. 93, No. 3 (Jun., 2003), pp. 530-550

[12] Grossman, Gene M. and Elhanan Helpman, "Quality Ladders in the Theory of Growth", Review of Economic Studies (1991) 58 (1): 43-61. 
[13] Haider, Steven J. "Earnings Instability and Earnings Inequality of Males in the United States: 1967-1991", Journal of Labor Economics, Vol. 19, No. 4. (Oct., 2001), pp. 799836 .

[14] Helpman, Elhanan and Manuel Trajtenberg, "Diffusion of General Purpose Technologies", in General Purpose Technologies and Economic Growth, Elhanan Helpman, editor. Cambridge, Mass.: MIT Press, 1998.

[15] Johansen L., "Substitution Versus Fixed Production Coeffcients in the Theory of Economic Growth", Econometrica 29,(1959) 157-176.

[16] Jovanovic, Boyan; Lach, Saul "Entry, Exit, and Diffusion with Learning by Doing" American Economic Review. September 1989; 79(4): 690-99.

[17] Jovanovic, Boyan and Peter L. Rousseau, "General Purpose Technologies", Handbook of Economic Growth, Volume1B, Philippe Aghion and Steven N. Durlauf, eds., Elsevier, 2005, pp. 1182-1221.

[18] Laitner, John P., Stolyarov, Dmitriy, "Aggregate Returns to Scale and Embodied Technical Change: Theory and Measurement", Journal of Monetary Economics, vol. 51, no 1 (Jan 2004): 191-233.

[19] Leahy, John V.; Zeira, Joseph "The Timing of Purchases and Aggragate Fluctuations", Review of Economic Studies, Volume 72, Number 4, October 2005, pp. 1127-1151.

[20] Mokyr, Joel, 1990a, "The lever of riches: Technological creativity and economic progress", Oxford; New York; Toronto and Melbourne: Oxford University Press, 1990.

[21] Mokyr, Joel, 1990b, "Twenty-five centuries of technological change: An historical survey", Fundamentals of Pure and Applied Economics series: Economics of Technological Change section. London; New York and Melbourne: Harwood Academic, 1990.

[22] Shapiro, Carl; Varian Hal R. Information Rules: A Strategic Guide to the Network Economy, Boston, Mass.: Harvard Business School Press, 1999.

[23] Shleifer, Andrei, "Implementation Cycles", The Journal of Political Economy, Vol. 94, No. 6. (Dec., 1986), pp. 1163-1190.

[24] Solow, Robert, "Investment and Technological progress" in Kenneth Arrow, Samuel Karlin and Patrick Suppes, eds. Mathematical Methods in the Social Sciences 1959. Stanford, CA: Stanford University Press, 1960: 89-104. 
[25] Swan, Peter L. "Optimum Durability, Second-Hand Markets, and Planned Obsolescence", The Journal of Political Economy, Vol. 80, No. 3, Part 1. (May - Jun., 1972), pp. 575-585. 


\section{Appendix: Proofs}

Proof of Proposition 1: If a consumer uses model $\tau-\alpha$ when the state-of-the-art model is $\tau$, her utility from durable consumption equals

$$
g(\tau-\alpha)=x_{\alpha}+g(\tau-T)
$$

Note that along the trajectory $(\alpha(t), c(t))$, the model being consumed at time $t$ is $\tau(t)=$ $\lfloor t\rfloor-\alpha(t)$, where $\lfloor t\rfloor$ denotes the integer part of $t$. Thus, the total discounted utility for the trajectory $(\alpha(t), c(t))$ is

$$
\begin{aligned}
U(\alpha, c) & =\int_{0}^{\infty} e^{-\rho t}\left[\ln \left(z_{\tau(t)}\right)+u(c(t))\right] d t=\int_{0}^{\infty} e^{-\rho t}[g(\lfloor t\rfloor-T+T-\alpha(t))+u(c(t))] d t \\
& =K+\int_{0}^{\infty} e^{-\rho t}\left[x_{\alpha(t)}+u(c(t))\right] d t,
\end{aligned}
$$

where

$$
K=\int_{0}^{\infty} e^{-\rho t} g(\lfloor t\rfloor-T) d t=\frac{g}{\rho}\left[\frac{\beta}{1-\beta}-T\right] .
$$

Arbitrarily, we can re-normalize utility to set $K=0$ without changing the consumer's preferences over consumption paths.

Proof of Theorem 1: Suppose that the agent is endowed with a durable of age $\alpha$ and follows an arbitrary purchasing policy $\tau=\left\{\tau_{k}\right\}_{k=1}^{\infty}$. We first show that the total cost and value $(b, v)$ of policy $\tau$ can be represented as a convex combination of the points $\left\{\left(b_{T, R}, v_{T, R}\right)\right\}_{R=1}^{T+1}$. Let $\tau_{0}=-\alpha$ and for all $k \geq 0$, let $r_{k}=\min \left\{\tau_{k+1}-\tau_{k}, T\right\}$ be the "holding time" for the durable purchased at time $\tau_{k}$. Then,

$$
b=p_{0} \sum_{k \geq 1} \beta^{\tau_{k}} \quad \text { and } \quad v=X_{\alpha, r_{0}}+\sum_{k \geq 1} \beta^{\tau_{k}} X_{0, r_{k}} .
$$

Define $K_{R}=\left\{k \geq 1 \mid r_{k}=R\right\}$ for $R=1, \ldots, T$ to be the set of purchases where the purchased durable is subsequently held for $R$ periods. Then,

$$
\beta^{\tau_{1}}=\left[\beta^{\tau_{1}}-\beta^{\tau_{2}}\right]+\left[\beta^{\tau_{2}}-\beta^{\tau_{3}}\right]+\cdots \geq \sum_{R=1}^{T} \sum_{k \in K_{R}} \beta^{\tau_{k}}\left(1-\beta^{R}\right),
$$

where the inequality is strict if for some $k \in K_{T}, \tau_{k+1}-\tau_{k}>T$. Let $\lambda_{R}=\sum_{K_{R}} \beta^{\tau_{k}}\left(1-\beta^{R}\right)$ for $R=1, \ldots, T$, and let $\lambda_{T+1}=1-\sum_{R=1}^{T} \lambda_{R}$. Thus, $\lambda_{R} \geq 0$ for all $R, \sum_{R=1}^{T+1} \lambda_{R}=1$, and, since $b_{T, T+1}=v_{T, T+1}=0$, 


$$
\begin{aligned}
& b=p_{0} \sum_{R=1}^{T} \sum_{k \in K_{R}} \beta^{\tau_{k}}=\sum_{R=1}^{T} \sum_{k \in K_{R}} \beta^{\tau_{k}}\left(1-\beta^{R}\right) b_{T, R}=\sum_{R=1}^{T+1} \lambda_{R} b_{T, R} \\
& v-X_{\alpha, r_{0}}=\sum_{R=1}^{T} X_{0, R} \sum_{k \in K_{R}} \beta^{\tau_{k}}=\sum_{R=1}^{T} \begin{array}{c}
X_{0, R} \\
1-\beta^{R}
\end{array} \sum_{k \in K_{R}} \beta^{\tau_{k}}\left(1-\beta^{R}\right)=\sum_{R=1}^{T+1} \lambda_{R} v_{T, R} .
\end{aligned}
$$

Put differently,

$$
\left[\begin{array}{c}
b \\
v-X_{\alpha, r_{0}}
\end{array}\right]=\sum_{R} \lambda_{R}\left[\begin{array}{c}
b_{T, R} \\
v_{T, R}
\end{array}\right]
$$

is a convex combination of the two-dimensional vectors $\left(b_{T, R}, v_{T, R}\right)$. Note that when $\alpha=T$, $X_{\alpha, r_{0}}=0$ for all $r_{0}{ }^{18}$

We next deduce an optimal policy for the case where $\alpha=T$ (i.e., when the agent is endowed with a useless durable). If $b \geq b_{T, 1}$, the agent can afford to replace the durable every period and $V_{T}(b)=v_{T, 1}$ (moreover, if $b>b_{T, 1}$, it is not possible for the agent to spend the budget $b$ in durables). For what follows, assume that $b<b_{T, 1}$. Let $R$ and $\lambda_{R}^{*} \in[0,1]$ be such that $b=\lambda_{R}^{*} b_{R}+\left(1-\lambda_{R}^{*}\right) b_{R+1}$. Since $\left(b, V_{T}(b)\right)=\sum \lambda_{R}\left(b_{T, R}, v_{T, R}\right)$ for some nonnegative weights $\lambda_{R}$ adding to 1 , we have that $V_{T}(b) \leq \lambda_{R}^{*} v_{T, R}+\left(1-\lambda_{R}^{*}\right) v_{T, R+1}$. To conclude, we need to only show that this bound is attained. For this, we need to show that there exists a policy $\tau$ such that $\sum_{k \in K_{R}} \beta^{\tau_{k}}\left(1-\beta^{R}\right)=\lambda_{R}^{*}$ and $\sum_{k \in K_{R+1}} \beta^{\tau_{k}}\left(1-\beta^{R+1}\right)=1-\lambda_{R}^{*}$. Put differently, we need to show that there exists an $R$-flexible rule with budget $b$.

Assume that $R<T$, and let $B_{R}^{*}$ denote the set of budgets $b(\tau)$ corresponding to policies $\tau$ that are $R$-flexible rules and satisfy $\tau_{1}=0$ (that is, $\tau$ makes a purchase in the first period). Let $\tau$ be such a policy and $\tau^{\prime}$ be its continuation policy from the period of the second purchase onward: $\tau_{t}^{\prime}=\tau_{t+1}-\tau_{1}$ for all $t \geq 1$. Then, $\tau^{\prime}$ is also an $R$-flexible rule, with $\tau_{1}^{\prime}=0$ and its corresponding budget $b\left(\tau^{\prime}\right) \in B_{R}^{*}$. Now, either $b(\tau)=p_{0}+\beta^{R} b\left(\tau^{\prime}\right)$ (if $\tau_{2}=R$ ) or $b(\tau)=p_{0}+\beta^{R+1} b\left(\tau^{\prime}\right)$ (if $\tau_{2}=R+1$ ). Therefore, $B_{R}^{*}$ is the largest set $B$ such that

$$
B=\left[p_{0}+\beta^{R} B\right] \cup\left[p_{0}+\beta^{R+1} B\right]
$$

Observe that $p_{0}+\beta^{R+1} b_{T, R+1}=b_{T, R+1}$ and $p_{0}+\beta^{R} b_{T, R}=b_{T, R}$, and that $p_{0}+\beta^{R} b_{T, R+1}<$ $p_{0}+\beta^{R+1} b_{T, R}$ when $\beta^{T-1}(1+\beta)>1$. Therefore, $B=\left[b_{T, R+1}, b_{T, R}\right]$ is a fixed point of (17). Since $p_{0}+\beta^{R} \tilde{b}<\tilde{b}$ for all $\tilde{b}>b_{T, R}$ and $p_{0}+\beta^{R+1} \tilde{b}>\tilde{b}$ for all $\tilde{b}<b_{T, R+1}, B$ is also the largest

\footnotetext{
${ }^{18}$ For each $R=1, \ldots, R$, we could define, instead, $L_{R}=\left\{\tau_{k} \mid k \in \mathbf{N}\right.$ and $\left.r_{k}=R\right\}$, as we did in Section 3. Then, $\lambda_{R}=\sum_{t \in L_{R}} \beta^{t}$. While $K_{R}$ contains the purchase numbers, $L_{R}$ contains the purchase dates (equivalently, model numbers) for the durables that are replaced at age $R$. However, for other purposes, the set $K_{R}$ is more convenient.
} 
such fixed point, and, thus, $B_{R}^{*}=B$. That is, for each budget $b \in B_{R}^{*}=\left[b_{T, R+1}, b_{T, R}\right]$, there exists an $(R, b)$-flexible rule (that spends the budget $b$ exactly). The proof for $R=T$ is similar (here, $b_{T, T+1}=0$, and we must consider policies $\tau$ where $\tau_{k+1}-\tau_{k}>T+1$ for some $k)$.

Finally, observe that if $(T, b)$ is the initial state, and $\tau$ and $\hat{\tau}$ are two $(R, b)$-flexible rules (they spend the same budget $b$ ), then their corresponding $\lambda_{R}$ (and $1-\lambda_{R}$ ) must coincide, and, therefore, they must have the same value, as well. In particular, if $b \in\left[b_{T, R+1}, b_{T, R}\right]$, then any $R$-flexible rule that spends the budget $b$ exactly is an optimal policy.

By construction, the value of following an $(R, b)$-flexible rule starting from a durable of age $T$ is given by

$$
V_{T}(b)=v_{T, R+1}+A_{R}\left(b-b_{T, R+1}\right), b \in\left[b_{T, R+1}, b_{T, R}\right], R=T, \ldots, 1 .
$$

When the endowed durable is of age $\alpha<T$, the corresponding optimal value function $V_{\alpha}(b)$ can be deduced from $V_{T}(b)$ from the observation that the continuation of an optimal policy is an optimal policy for the subproblem that arises in the second period after following the policy in the first period.

If - starting with a budget $b \in\left[p_{0}, b_{T, 1}\right]=\left[b_{T+1, T+1}, b_{T, 1}\right]$ - a consumer buys a durable in the first period and then keeps it for the next $\alpha-1$ periods, her budget at the beginning of period $\alpha \geq 1$ is $\theta_{\alpha}(b)=\left(b-p_{0}\right) / \beta^{\alpha}$. Moreover, for any $1 \leq R \leq T+1$ and $1 \leq \alpha \leq \min \{R, T\}$, $\theta_{\alpha}\left(b_{T, R}\right)=b_{\alpha, R}$.

Assume that the initial state is $(\alpha, b)$, where $1 \leq \alpha<T$ and $b \in\left[b_{\alpha, R+1}, b_{\alpha, R}\right]$ for some $\alpha \leq R \leq T$. Let $\tilde{b}=p_{0}+\beta^{\alpha} b$. Then, $b=\theta_{\alpha}(\tilde{b})$. Since $b \in\left[b_{\alpha, R+1}, b_{\alpha, R}\right]$, it must be that $\tilde{b} \in\left[b_{T, R+1}, b_{T, R}\right]$. Therefore, starting at state $(T, \tilde{b})$, it is optimal to follow an $R$-flexible rule. Assume that the consumer does so. Then, after $\alpha$ periods, her state becomes $(\alpha, b)$, and from state $(\alpha, b)$ she must be following an $R$-flexible rule, as well. Hence, the agent must keep the durable for another $R-\alpha$ periods (at least). At that point, she arrives at state $\left(R, b / \beta^{R-\alpha}\right)$. Note that $\left(1 / \beta^{R-\alpha}\right)\left[b_{\alpha, R+1}, b_{\alpha, R}\right]=\left[b_{R, R+1}, b_{R, R}\right]$ and that $\beta^{R-\alpha} b_{R+1, R+1}=b_{\alpha+1, R+1} \in\left(b_{\alpha, R+1}, b_{\alpha, R}\right)$. Hence, if $b / \beta^{R-\alpha} \in\left[b_{R, R+1}, b_{R+1, R+1}\right)$, she must keep the durable this period and buy a new durable next period, so her continuation value is $\hat{x}_{R}+V_{T}\left(b / \beta^{R+1-\alpha}\right)$. If $b / \beta^{R-\alpha} \in\left[b_{R+1, R+1}, b_{R, R}\right]$, she can optimally buy a new durable this period, and her continuation value is $V_{T}\left(b / \beta^{R-\alpha}\right)$. Therefore,

$$
V_{\alpha}(b)= \begin{cases}X_{\alpha, R+1}+\beta^{R+1-\alpha} V_{T}\left(b / \beta^{R+1-\alpha}\right) & \text { for } b \in\left[b_{\alpha, R+1}, b_{\alpha+1, R+1}\right) \\ X_{\alpha, R}+\beta^{R-\alpha} V_{T}\left(b / \beta^{R-\alpha}\right) & \text { for } b \in\left[b_{\alpha+1, R+1}, b_{\alpha, R}\right] .\end{cases}
$$

Suppose that $b \in\left[b_{\alpha, R+1}, b_{\alpha+1, R+1}\right)$. Then $b / \beta^{R+1-\alpha} \in\left[b_{R+1, R+1}, b_{R+1, R+1} / \beta\right) \subset\left[b_{T, R+1}, b_{T, R}\right]$. 
Therefore, $V_{T}\left(b / \beta^{R+1-\alpha}\right)=v_{T, R+1}+A_{R}\left(b / \beta^{R+1-\alpha}-b_{T, R+1}\right)$, and

$$
X_{\alpha, R+1}+\beta^{R+1-\alpha} V_{T}\left(b / \beta^{R+1-\alpha}\right)=v_{\alpha, R+1}+A_{R}\left(b-b_{\alpha, R+1}\right) .
$$

Now, suppose that $b \in\left[b_{\alpha+1, R+1}, b_{\alpha, R}\right]$. Then, $b / \beta^{R-\alpha} \in\left[b_{R, R+1}, b_{R, R} / \beta\right) \subset\left[b_{T, R+1}, b_{T, R}\right]$. Therefore, $V_{T}\left(b / \beta^{R-\alpha}\right)=v_{T, R+1}+A_{R}\left(b / \beta^{R-\alpha}-b_{T, R+1}\right)$, and tedious algebra shows again that

$$
X_{\alpha, R}+\beta^{R-\alpha} V_{T}\left(b / \beta^{R-\alpha}\right)=v_{\alpha, R+1}+A_{R}\left(b-b_{\alpha, R+1}\right) .
$$

Therefore, for all $\alpha \leq R \leq T$ and $b \in\left[b_{\alpha, R+1}, b_{\alpha, R}\right], V_{\alpha}(b)=v_{\alpha, R+1}+A_{R}\left(b-b_{\alpha, R+1}\right)$. It remains to find $V_{\alpha}(b)$ for $b>b_{\alpha, \alpha}$. We claim that $V_{\alpha}(b)=V_{T}(b)$ for all $b>b_{\alpha, \alpha}$. Since $b_{\alpha, R}=b_{T, R}$ for all $R \leq \alpha$, we have that $V_{T}(b)=v_{\alpha, R+1}+A_{R}\left(b-b_{\alpha, R+1}\right)$ for all $b \in\left[b_{\alpha, R+1}, b_{\alpha, R}\right]$ and $1 \leq R<\alpha$, and the claim would complete the proof. To prove our claim, we show that

$$
V_{T}(b)>X_{\alpha, s+\alpha}+\beta^{s} V_{T}\left(b / \beta^{s}\right) \text { for all } s>0 \text { and } b>b_{\alpha, \alpha} .
$$

That is, when $b>b_{\alpha, \alpha}$, the consumer strictly prefers to replace the durable immediately rather than replacing it at any later time. One can check that the above inequality holds when $b=b_{\alpha, \alpha}$. Also, since $V_{T}$ is concave, the function $V_{T}(b)$ has a higher slope than the function on the right-hand side for any $b>0$. Hence, the inequality holds for every $b>b_{\alpha, \alpha}$.

Proof of Theorem 2: Consider an arbitrary purchasing policy $\left\{\left(\tau_{k}^{\prime}, d_{k}\right)\right\}_{k=1}^{\infty}$, where $\tau_{k}^{\prime}+d_{k}$ denotes the time of the $k$-th purchase and $\tau_{k}^{\prime} \in \mathbf{N}$ its corresponding period (so $d_{k} \in[0,1)$ denotes its "delay"). Let $r_{0}=\alpha+\tau_{1}^{\prime}$, and for all $k \geq 1$, let $\tau_{k}=\tau_{k}^{\prime}-\tau_{1}^{\prime}$ and $r_{k}=\min \left\{\tau_{k+1}-\tau_{k}, T\right\}$. Then, the continuation budget and value of such a policy, at the beginning of period $\tau_{1}^{\prime}$, are, respectively,

$$
\begin{aligned}
b & =p_{0} \sum_{k \geq 1} \beta^{\tau_{k}} e^{-\rho d_{k}}=p_{0} \sum_{k \geq 1} \beta^{\tau_{k}}-p_{0} \sum_{k \geq 1} \beta^{\tau_{k}}\left(1-e^{-\rho d_{k}}\right) \\
v & =\sum_{k \geq 1} \beta^{\tau_{k}} X_{0, r_{k}}-\sum_{k \geq 1} \beta^{\tau_{k}}\left(x_{0}-x_{r_{k-1}}\right)\left(1-e^{-\rho d_{k}}\right) / \rho .
\end{aligned}
$$

Let $I_{r_{0}}=1$ and $I_{R}=0$ for $R \neq r_{0}$. For $1 \leq R \leq T$, let $K_{R}=\left\{k \geq 1 \mid r_{k}=R\right\}$,

$$
\begin{aligned}
& \lambda_{R}=\sum_{k \in K_{R}} \beta^{\tau_{k}}\left(1-\beta^{R}\right), \quad \bar{\gamma}_{R}=I_{R} \beta^{0}+\sum_{k \in K_{R}} \beta^{\tau_{k+1}} \quad \text { and } \\
& \gamma_{R}=I_{R} \beta^{0}\left[\frac{1-e^{-\rho d_{1}}}{1-\beta}\right]+\sum_{k \in K_{R}} \beta^{\tau_{k+1}}\left[\frac{1-e^{-\rho d_{k+1}}}{1-\beta}\right]
\end{aligned}
$$


so that

$$
\left[\begin{array}{l}
b \\
v
\end{array}\right]=\left[\begin{array}{l}
\hat{b} \\
\hat{v}
\end{array}\right]-\sum_{R=1}^{T} \gamma_{R}\left[\begin{array}{c}
p_{0}(1-\beta) \\
\hat{x}_{0}-\hat{x}_{R}
\end{array}\right], \quad \text { where } \quad\left[\begin{array}{l}
\hat{b} \\
\hat{v}
\end{array}\right]=\sum_{R=1}^{T} \lambda_{R}\left[\begin{array}{c}
b_{T, R} \\
v_{T, R}
\end{array}\right] .
$$

The coefficient $\lambda_{R}$ incorporates the discounting of all the periods in which a purchase is made for a good that will be replaced at age $R$. By contrast, $\gamma_{R}$ incorporates the discounting of all the periods in which a purchase is made to replace a good of age $R$. The adjustment, reflected in the factor multiplying $I_{r_{0}}$, of $\gamma_{r_{0}}$ (and $\bar{\gamma}_{r_{0}}$ ) is required to take into account the first purchase that replaces the endowed good (which, in our accounting, was not previously purchased). Observe that for $1 \leq R \leq T-1, k \in K_{R}$ implies that $\tau_{k+1}=\tau_{k}+R$ (if $k \in R_{T}$ then $\tau_{k+1} \geq \tau_{k}+T$, where strict inequality holds when a useless good is not replaced for one or more periods). Therefore,

$$
\begin{aligned}
& \bar{\gamma}_{R}=I_{R}+\left[\frac{\beta^{R}}{1-\beta^{R}}\right] \lambda_{R} \text { for all } 1 \leq R \leq T-1, \text { and } \\
& \sum_{R=1}^{T} \frac{\lambda_{R}}{1-\beta^{R}}=\sum_{k \geq 1} \beta^{\tau_{k}}=\sum_{R=1}^{T} \bar{\gamma}_{R}=\sum_{R=1}^{T-1}\left[\begin{array}{c}
\beta^{R} \\
1-\beta^{R}
\end{array} \lambda_{R}+I_{R}\right]+\bar{\gamma}_{T} .
\end{aligned}
$$

Hence, $\bar{\gamma}_{T}=\sum_{R=1}^{T-1} \lambda_{R}+\lambda_{T} /\left(1-\beta^{T}\right)+I_{T}-1=I_{T}+\lambda_{T} \beta^{T} /\left(1-\beta^{T}\right)$. Let $\Lambda=\left\{\lambda \in \mathbf{R}_{+}^{T} \mid\right.$ $\left.\sum_{R=1}^{T} \lambda_{R} \leq 1\right\}$, and

$$
\Gamma=\left\{(\lambda, \gamma) \in \Lambda \times \mathbf{R}_{+}^{T} \mid \gamma_{R}<\bar{\gamma}_{R} \quad \text { for } 1 \leq R \leq T\right\}
$$

The argument above essentially contains the proof of the following claim.

CLAIM 1: Let $\left\{\left(\tau_{k}^{\prime}, d_{k}\right)\right\}$ be an arbitrary purchasing policy and $(\lambda, \gamma)$ be the weights defined by (18). Then, $(\lambda, \gamma) \in \Gamma$. Conversely, for any $(\lambda, \gamma) \in \Gamma$ (and $\tau_{1}^{\prime} \geq 1$ ), there exists a purchasing policy $\left\{\left(\tau_{k}^{\prime}, d_{k}\right)\right\}$ that satisfies (18). Though this policy is usually not unique, all such policies have the same budget and value. Thus, with abuse of notation, we will also refer to a $(\lambda, \gamma) \in \Gamma$ as a purchasing policy.

CLAIM 2: Suppose that the policy corresponds to an $R$-flexible rule where $\tau_{1}=0$, and the replacement of durables of age $R+1$ is never delayed, but the replacement of durables of age $R$ is sometimes delayed. Then, the policy is suboptimal: there exists another $R$-flexible rule without delays that costs the same and has a strictly higher value.

To prove claim 2, observe that for such a policy, $\lambda_{R}+\lambda_{R+1}=1, \gamma_{R}>0, \gamma_{R+1}=0$, and $\lambda_{k}=\gamma_{k}=0$ for all $k \notin\{R, R+1\}$. Moreover, since $\gamma_{R}<\lambda_{R} \beta^{R} /\left(1-\beta^{R}\right)$, we also have $\lambda_{R}>0$. In this case, $(\hat{b}, \hat{v})$ is on the "Pareto frontier" (i.e., $\hat{v}=V_{T}(\hat{b})$ ). The vector 
$(\hat{b}, \hat{v})-(b, v)=\left(p_{0}(1-\beta), \hat{x}_{0}-\hat{x}_{R}\right)$ has "slope" $\sigma=\left[\hat{x}_{0}-\hat{x}_{R}\right] /\left[p_{0}(1-\beta)\right]$, and

$$
A_{R}=\frac{1}{p_{0}}\left[X_{0, R}-\hat{x}_{R} \frac{1-\beta^{R}}{1-\beta}\right] \leq\left(1-\beta^{R}\right) \frac{\hat{x}_{0}-\hat{x}_{R}}{p_{0}(1-\beta)}<\sigma .
$$

So, as the delays increase $\left(\gamma_{R}\right.$ increases $),(b, v)$ moves away from $(\hat{b}, \hat{v})$, below the Pareto frontier. But, if $\sigma<A_{R+1}$, the delays may eventually take $(b, v)$ back above the Pareto frontier. This can happen only if $b<b_{T, R+1}$. But even if every durable of age $R$ is replaced with delay, the cost of the policy is more than replacing the durables at age $R+1$ all the time. That is, $b \geq b_{T, R+1}$. Therefore, $b_{T, R+1} \leq b \leq b_{T, R}$ and $v<V_{T}(b)$, and there exists another $R$-flexible rule with no delays that costs $b$ and has value $V_{T}(b)$.

CLAIM 3: Suppose that the policy $\left\{\left(\tau_{k}, d_{k}\right)\right\}$ is such that $\gamma_{R}>0$ for some $R$. Then, the policy is suboptimal: there exists another policy without delays that uses the same budget but has strictly higher value.

Assume that the policy has delays. We now recursively modify the policy by eliminating delays, while maintaining the same budget and improving its value in every step. Let $h=$ $\lambda_{1}+\lambda_{2}, \hat{\lambda}_{k}=\lambda_{k} / h$ for $k=1,2$, and $\hat{\gamma}_{1}=\gamma_{1} / h$. Then,

$$
\left[\begin{array}{l}
b \\
v
\end{array}\right]=h\left(\hat{\lambda}_{1}\left[\begin{array}{l}
b_{T, 1} \\
v_{T, 1}
\end{array}\right]+\hat{\lambda}_{2}\left[\begin{array}{c}
b_{T, 2} \\
v_{T, 2}
\end{array}\right]-\hat{\gamma}_{1}\left[\begin{array}{c}
p_{0}(1-\beta) \\
\hat{x}_{0}-\hat{x}_{1}
\end{array}\right]\right)+\sum_{R=2}^{T}\left(\lambda_{R}\left[\begin{array}{c}
b_{T, R} \\
v_{T, R}
\end{array}\right]-\gamma_{R}\left[\begin{array}{c}
p_{0}(1-\beta) \\
\hat{x}_{0}-\hat{x}_{R}
\end{array}\right]\right) .
$$

The weights $\left(\hat{\lambda}_{1}, \hat{\lambda}_{2}, \hat{\gamma}_{1}\right)$ represent a 1 -flexible rule with delays (and $\left.\hat{\lambda}_{1}+\hat{\lambda}_{2}=1\right)$. If $\gamma_{1}>0$ (so $\hat{\gamma}_{1}>0$ ), then, by Claim 3 , there exists another 1 -flexible rule with weights $\left(\tilde{\lambda}_{1}, \tilde{\lambda}_{2}, 0\right)$ that is better. Let $\lambda_{k}^{\prime}=h \tilde{\lambda}_{k}$ for $k=1,2, \gamma_{1}^{\prime}=0, \lambda_{k}^{\prime}=\lambda_{k}$ for $k \geq 3$, and $\gamma_{k}^{\prime}=\gamma_{k}$ for $k \geq 2$. The policy $\left(\lambda^{\prime}, \gamma^{\prime}\right)$ is better than the policy $(\lambda, \gamma)$ and has $\gamma_{1}^{\prime}=0$. Now, let $h=\lambda_{2}^{\prime}+\lambda_{3}^{\prime}, \hat{\lambda}_{k}=\lambda_{k}^{\prime} / h$ for $k=2,3$, and $\hat{\gamma}_{2}=\gamma_{2}^{\prime} / h$. The weights $\left(\hat{\lambda}_{2}, \hat{\lambda}_{3}, \hat{\gamma}_{2}\right)$ represent a 2 -flexible rule with delays. Again, if $\hat{\gamma}_{2}>0$, Claim 3 implies that there exists a better 2-flexible rule without delays that can be used to modify $\left(\lambda^{\prime}, \gamma^{\prime}\right)$ and construct a new policy $\left(\lambda^{\prime \prime}, \gamma^{\prime \prime}\right)$ that uses the same budget and improves the value, and has $\gamma_{1}^{\prime \prime}=\gamma_{2}^{\prime \prime}=0$. Continuing this way, after $T$ steps, we will have constructed a policy $\left(\lambda^{*}, \gamma^{*}\right)$ with $\gamma^{*}=0$, which uses the same budget and has a higher value than $(\lambda, \gamma)$.

Finally, by Claim 2 (or Theorem 1), for any $\tau_{1}^{\prime} \geq 1$ and any weights $\lambda^{*}$, there exist $R$ and an $R$-flexible rule that uses the same budget $\beta^{\tau_{1}^{\prime}} b=\beta^{\tau_{1}^{\prime}} \sum_{k} \lambda_{k}^{*} b_{T, k}$ (from period 0 onward) and delivers a (weakly) better value. Therefore, the optimal value function $V_{T}$ coincides with that in expression (9), as defined in Theorem 1.

Proof of Theorem 3: Let $c=(1-\beta)(w-b)$, so that $b=w-c /(1-\beta)$. With this 
change of variables, problem (4) becomes

$$
U_{\alpha}(w)=\max _{c \in[0,(1-\beta) w]} \frac{\hat{u}(c)}{1-\beta}+V_{\alpha}(w-c /(1-\beta)) .
$$

Let $B(w, c)=w-c /(1-\beta)$. Note that the objective function $\varphi(c)=\hat{u}(c) /(1-\beta)+$ $V_{\alpha}(B(w, c))$ is concave. Thus, $\hat{c}$ maximizes $\varphi(c)$ if and only if $0 \in \partial \varphi(\hat{c})$ (that is, 0 is a subdifferential of $\varphi$ at $\hat{c})$ or equivalently, if and only if $\hat{u}^{\prime}(\hat{c}) \in \partial V_{\alpha}(B(w, \hat{c}))$. There are two cases: (1) $V_{\alpha}$ is differentiable at $B(w, \hat{c})$; and (2) $V_{\alpha}$ has a kink at $B(w, \hat{c})$.

Case 1: Observe that $B\left(w, c_{R}\right) \in\left(b_{\alpha, R+1}, b_{\alpha, R}\right)$ if and only if $w \in\left(w_{\alpha, R+1}\left(c_{R}\right), w_{\alpha, R}\left(c_{R}\right)\right)$. Now, if $B\left(w, c_{R}\right) \in\left(b_{\alpha, R+1}, b_{\alpha, R}\right)$ for some $R$, then $\hat{u}^{\prime}\left(c_{R}\right)=A_{R}=V_{\alpha}^{\prime}\left(B\left(w, c_{R}\right)\right)$, and $c_{R}$ is the optimal solution of problem (4). That is, when $w \in\left(w_{\alpha, R+1}\left(c_{R}\right), w_{\alpha, R}\left(c_{R}\right)\right)$, it is optimal to consume a constant flow $c_{R}$ of non-durables and follow an $R$-flexible rule for the durable good. One can check that $B\left(w, c_{\alpha}\right)=b_{\alpha+1, \alpha+1} \Leftrightarrow w=w_{\alpha+1, \alpha+1}\left(c_{\alpha}\right)$ and $B\left(w, c_{\alpha}\right)=b_{\alpha-1, \alpha} \Leftrightarrow$ $w=w_{\alpha-1, \alpha}\left(c_{\alpha}\right)$, and $w_{\alpha, \alpha+1}\left(c_{\alpha}\right)<w_{\alpha+1, \alpha+1}\left(c_{\alpha}\right)<w_{\alpha-1, \alpha}\left(c_{\alpha}\right)<w_{\alpha, \alpha}\left(c_{\alpha}\right)$. Therefore, $\delta_{\alpha}^{*}(w)$ is given by (13).

Case 2: Observe that $A_{R-1} \leq \hat{u}^{\prime}\left(c_{\alpha, R}(w)\right) \leq A_{R}$ if and only if $c_{R} \leq c_{\alpha, R}(w) \leq c_{R-1}$, or, alternatively, if and only if $w \in\left[w_{\alpha, R}\left(c_{R}\right), w_{\alpha, R}\left(c_{R-1}\right)\right]$. Since $B\left(w, c_{\alpha, R}(w)\right)=b_{\alpha, R}$ and $\partial V_{\alpha}\left(b_{\alpha, R}\right)=\left[A_{R-1}, A_{R}\right]$, if $\hat{u}^{\prime}\left(c_{\alpha, R}(w)\right) \in\left[A_{R-1}, A_{R}\right]$ for some $R$, then $c_{\alpha, R}(w)$ is the optimal solution of problem (4). That is, it is optimal to consume a constant flow $c_{\alpha, R}(w)$ of non-durables and follow the $R$-fixed purchasing rule for the durable good. In particular, $\delta_{\alpha}^{*}(w)=1$ if $R \leq \alpha$ (or, equivalently, if $w \geq w_{\alpha, \alpha}\left(c_{\alpha}\right)$ ) and $\delta_{\alpha}^{*}(w)=0$ if $R>\alpha$, as stated in (13).

For a fixed $\alpha$, the intervals corresponding to case 1 alternate with those corresponding to case 2. Moreover, collectively, they are mutually exclusive and cover the whole wealth range.

Proof of Proposition 2: As argued in the text, gains from trade within the 1-flexible class are zero, leaving the 1-fixed class as the only candidate sellers of used goods. The proof proceeds in three steps.

Step 1 Derive the upper bound on the used-good price acceptable to the buyer. A buyer is someone with $\alpha=1$, budget $b<b_{11}, V_{1}(b)=A_{1} b$ and optimal non-durable consumption $c_{1}$. Instead of purchasing a new good right away and following a 1-flexible rule afterwards, this consumer can purchase a used good of age $s$ at price $p(s)$ and follow a 1-flexible rule forever after. The flexible rule prescribes that the gain from trade is spent exclusively on 
durables. Hence, the buyer's lifetime utility from a used good purchased at age $s$ is

$$
U_{B}=\underbrace{e^{-\rho s} X_{s, 1}}_{\text {Durable service, this period }}+\underbrace{\beta V_{1}\left(\frac{b-p(s) e^{-\rho s}}{\beta}\right)}_{\text {Durable service, future }}+\frac{1}{1-\beta} \hat{u}\left(c_{1}\right) .
$$

With no trade, her utility is

$$
U_{N}=A_{1} b+\frac{1}{1-\beta} \hat{u}\left(c_{1}\right)
$$

The gain from trade for the buyer is

$$
U_{B}-U_{N}=e^{-\rho s}\left[X_{s, 1}-A_{1} p(s)\right] \geq 0
$$

Step 2 A good $x_{0}$ that is sold in the middle of the period is not going to be replaced with a new good until the end of the period. Indeed, a potential seller has three options: keep the good for one period; sell in the middle of the period and replace with a new good, or sell and not replace until the next model. "Keep" always dominates "sell and replace" during the first period since $p(s)<p_{0}$. Then, the only trading strategy that involves selling in the middle of the period has the seller without a good for the rest of the period.

Step 3 Any used-goods seller will find the price $p(s)$ unacceptably low. To see this, take a 1-fixed rule consumer whose wealth is $w \geq w_{11}$. Theorem 3 shows that $\hat{u}^{\prime}\left(c_{1}^{*}(w)\right) \leq A_{1}$ for the entire 1-fixed-rule group. The payoff from following the 1-fixed rule is

$$
U_{1}=\frac{x_{0}}{\rho}+\frac{1}{1-\beta} \hat{u}(c) .
$$

If a 1-fixed consumer sells a used good, she will spend the gain from trade exclusively on non-durables. Specifically, if the trade brings a gain in wealth of size $\Delta w$, the non-durable consumption will increase by $\Delta c=(1-\beta) \Delta w$. Therefore, the payoff from selling the used good at age $s$ and following a 1-fixed rule forever after is

$$
\begin{gathered}
U_{S}=\frac{x_{0}}{\rho}-e^{-\rho s} X_{s, 1}+\frac{1}{1-\beta} \hat{u}(c+\Delta c)<[\hat{u} \text { is concave }] \\
<\frac{x_{0}}{\rho}+\frac{1}{1-\beta} \hat{u}(c)-e^{-\rho s} X_{s, 1}+\frac{1}{1-\beta} \hat{u}^{\prime}(c) \Delta c \leq \\
\leq U_{1}-e^{-\rho s} X_{s, 1}+A_{1} \cdot e^{-\rho s} \cdot p(s)=U_{1}+U_{N}-U_{B}
\end{gathered}
$$

The last inequality establishes that gains from trade are non-positive.

Proof of Proposition 3: We first construct an upper bound $W(\bar{b})$ for $V_{1}(\bar{b}, 0)$. Let $d$ be 
such that $e^{\rho d} \bar{b}=1$. The agent has to wait at least until $d$ before she can afford to purchase a new durable. If the agent consumes an old durable until $d$ and a new durable forever after, her total discounted value is

$$
W(\bar{b})=\frac{x_{1}}{\rho}\left(1-e^{-\rho d}\right)+\frac{x_{0}}{\rho} e^{-\rho d}=\frac{x_{1}}{\rho}(1-\bar{b})+\frac{x_{0}}{\rho} \bar{b} .
$$

Clearly, $V_{1}(\bar{b}, 0) \leq W(\bar{b})$ for all $\bar{b} \in[0,1)$. Also, $V_{1}(0,0)=W(0)=x_{1} / \rho$. Therefore,

$$
\frac{\partial}{\partial \bar{b}} V_{1}(0,0) \leq W^{\prime}(0)=\frac{x_{0}-x_{1}}{\rho}
$$

Differentiating both sides of the Bellman equation (14) with respect to $\bar{b}$ and evaluating the derivative at $\bar{b}=0$ gives

$$
\frac{\partial}{\partial \bar{b}} V_{0}(0, S)=\frac{\partial}{\partial \bar{b}} V_{1}(0,0) .
$$

For convenience, let $G(\bar{b}, d)$ denote the right-hand side of (15). This function is defined for all $(\bar{b}, d)$ where a purchase with delay $d \leq S$ is feasible - that is, for $\bar{b} \geq e^{-(r+\gamma) S}$ and $\max \left\{0, \frac{1}{r+\gamma} \ln \left(\frac{1}{b}\right)\right\} \leq d \leq S$. Now, we show that when $\rho=r+\gamma, G(\bar{b}, d)$ is strictly decreasing in $d$ for all $\bar{b} \geq e^{-(r+\gamma) S}$. Differentiating $G$ with respect to $d$ and setting $\rho=r+\gamma$, we get

$$
\begin{aligned}
\frac{\partial G}{\partial d}(\bar{b}, d) & =e^{-\rho d} \rho\left[\frac{\partial}{\partial \bar{b}} V_{0}\left(e^{\rho S}\left(\bar{b}-e^{-\rho d}\right), S\right)-\frac{\left(x_{0}-x_{1}\right)}{\rho}\right] \\
& <e^{-\rho d} \rho\left[\frac{\partial}{\partial \bar{b}} V_{0}(0, S)-\frac{\left(x_{0}-x_{1}\right)}{\rho}\right] \leq 0 .
\end{aligned}
$$

If $\bar{b} \geq 1$, any delay $d \in[0, S]$ is possible. Since $G(\bar{b}, d)$ is strictly decreasing in $d$ for all $d \in[0, S]$, the optimal delay must be either $d=0$ or $d>S$.

If $\bar{b}<1$, delays $d<\frac{1}{r+\gamma} \ln (1 / \bar{b})$ are not feasible, but since $G(\bar{b}, d)$ is decreasing in $d$, the maximum is either $d(\bar{b})=\frac{1}{r+\gamma} \ln (1 / \bar{b})<S$ or $d(\bar{b})>S$. 
Figure 1: Optimal value function $V_{1}(b)$ and optimal decision rules $\delta_{1}^{*}(b)$ and $c_{1}^{*}(b)$, special case $T=1$.

Figure 2: Optimal value function, general case. 
Figure 3: The first-order condition for optimal consumption (left) and the optimal decision rule $c_{\alpha}^{*}(w)$.

Figure 4: A diffusion curve with an adoption plateau. 
Figure 5: No-purchase intervals for various values of $\gamma, \lambda$ and $S$. 